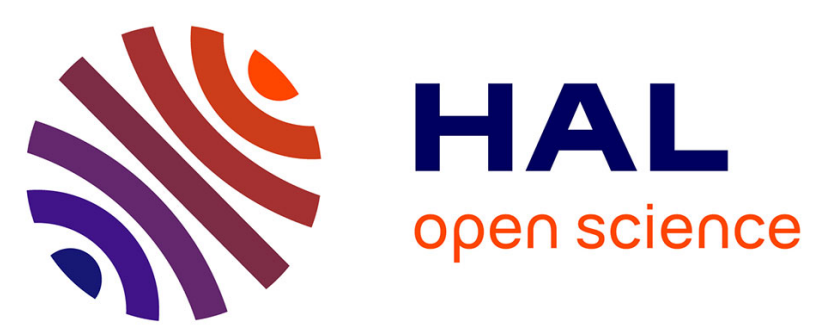

\title{
Boundary layer method for solving full Maxwell equations in the presence of an electromagnetic inhomogeneity of small diameter
}

Abdessatar Khelifi, Saoussen Boujemaa

\section{- To cite this version:}

Abdessatar Khelifi, Saoussen Boujemaa. Boundary layer method for solving full Maxwell equations in the presence of an electromagnetic inhomogeneity of small diameter. 2021. hal-01465066v2

\section{HAL Id: hal-01465066 \\ https://hal.science/hal-01465066v2}

Preprint submitted on 1 Mar 2021

HAL is a multi-disciplinary open access archive for the deposit and dissemination of scientific research documents, whether they are published or not. The documents may come from teaching and research institutions in France or abroad, or from public or private research centers.
L'archive ouverte pluridisciplinaire HAL, est destinée au dépôt et à la diffusion de documents scientifiques de niveau recherche, publiés ou non, émanant des établissements d'enseignement et de recherche français ou étrangers, des laboratoires publics ou privés. 


\title{
Boundary layer method for solving full Maxwell equations in the presence of an electromagnetic inhomogeneity of small diameter
}

\author{
Abdessatar Khelifi * $\quad$ Saoussen Boujemaa $^{\dagger}$
}

February 27, 2021

\begin{abstract}
We consider solutions to the time-harmonic Maxwell's equations in two and three dimensions. For such solutions we derive high-order terms in the asymptotic expansions of the perturbations resulting from the presence of diametrically small electromagnetic inhomogeneity with parameters different from the background medium. Our study is rigorous and is founded on layer potential techniques. Our formulas may be awaited to head effective computational identification algorithms, aimed at reconstructing small dielectric object from electromagnetic boundary measurements.
\end{abstract}

Mathematics subject classification (MSC2010): 35Q60, 35B30, 35B40

Keywords: Full Maxwell's equations, electromagnetic inhomogeneity, electromagnetic potentials, asymptotic formula.

\section{Introduction}

In this paper, we use layer potential techniques to derive complete asymptotic expansions for (tangential) boundary perturbations in the solutions of the Maxwell equations resulting from small perturbations of the shape of an inhomogeneity with smooth boundary in $\mathbb{R}^{d}$, for $d=2,3$. These formulae may permit us to advance the development of high-order terms in the asymptotic expansions of the boundary perturbations of electromagnetic fields caused by the presence of small inhomogeneity. So at this stage, a development of effective algorithms for determining certain properties of the shape of an inhomogeneity will be more possible. Moreover, the

*Département de Mathématiques, Faculté des Sciences de Bizerte, Université de Carthage, Tunisia. (Email: abdessatar.khelifi@fsb.rnu.tn)

${ }^{\dagger}$ Département de Mathématiques, Faculté des Sciences de Bizerte, Université de Carthage, Tunisia. (Email: soussenboujemaa2013@gmail.com). 
general approach of this paper can be extended to other investigations such as the context of interface problems related to electromagnetic boundary measurements, and to develop complete and precise asymptotic expansions of both eigenvalues and eigenfunction associated to full Maxwell equations as a generalization of the previous results. Thus, it will be easy to provide accurate energy estimates and reconstruction procedures of interfaces from measurements of these eigenvalues and eigenfunctions. Time-harmonic Maxwell equations occur in many applications and frequently involve non homogeneous domains, making boundary integral formulation advantageous. But, compared with other elliptic problems such as the Helmholtz equation Maxwell equations pose supplementary difficulties in boundary conditions. More precisely, boundary conditions are formulated in terms of tangential components of magnetic and of electric fields. In fact, this difficulty requires spaces so that traces and operators defined by integral equations are continuous, leading in a natural way to variational formulations in specific Hilbert spaces. Beyond electric field integral equations, to the best of our knowledge, no theory existed for boundary layer techniques related to Maxwell equations by providing complete asymptotic expansions of solutions with respect to shape variation parameter.

Let $\Omega$ be a bounded domain in $\mathbb{R}^{d}, d=2$ or 3 , with a connected Lipschitz boundary $\partial \Omega$. Let $\nu$ denote the unit outward normal to $\partial \Omega$. Suppose that $\Omega$ contains a small inhomogeneity $D$ of the form $D=z+\delta B$ where $B$, is a bounded Lipschitz domain in $\mathbb{R}^{d}$ containing the origin, and $\delta$ is the order of magnitude of the diameter of the inhomogeneity. We assume that the domain $D$ is separated apart from the boundary. More precisely, we assume that there exists a constant $c_{0}>0$ such that:

$$
\operatorname{dist}(z, \partial \Omega) \geq c_{0}>0
$$

Let $\varepsilon^{r e}$ be the (real) electric permittivity and $\mu$ the magnetic permeability of the domain $\Omega$ containing different materials. The time-dependent linear Maxwell equations in $\Omega$ take the form:

$$
\left\{\begin{array}{c}
\nabla \times \mathcal{E}(x, t)=-\mu(x) \partial_{t} \mathcal{H}(x, t), \quad \text { for }(x, t) \in \Omega \times(0, \infty), \\
\nabla \times \mathcal{H}(x, t)=\varepsilon^{r e}(x) \partial_{t} \mathcal{E}(x, t)+\mathbf{J}_{f}(x, t), \quad \text { for }(x, t) \in \Omega \times(0, \infty),
\end{array}\right.
$$

where $\mathcal{E} \in \mathbb{C}^{d}$ is the electric field and $\mathcal{H} \in \mathbb{C}^{d}$ is the magnetic field. But, $\mathbf{J}_{f}$ is the free current related to the field $\mathcal{E}$ by $\mathbf{J}_{f}=\sigma \mathcal{E}$, where $\sigma$ represents the conductivity of the medium.

In this paper we study the time-harmonic solutions to these equations, we consider special solutions of the form:

$$
\mathcal{E}(x, t)=\Re\left(\mathbf{E}(x) e^{-i \omega t}\right) \quad \text { and } \mathcal{H}(x, t)=\Re\left(\mathbf{H}(x) e^{-i \omega t}\right), \quad x \in \Omega, t>0,
$$

where $\omega>0$ is a given frequency, and where the $\mathbb{C}^{d}$ valued fields $\mathbf{E}(\mathbf{x})$ and $\mathbf{H}(\mathbf{x})$ 
satisfy the time-harmonic Maxwell equations:

$$
\left\{\begin{array}{l}
\nabla \times \mathbf{E}=i \omega \mu \mathbf{H} \quad \text { in } \Omega, \\
\nabla \times \mathbf{H}=-i \omega \varepsilon \mathbf{E} \quad \text { in } \Omega,
\end{array}\right.
$$

where $\varepsilon(x)=\varepsilon^{r e}(x)+i \frac{\sigma(x)}{\omega}$, for $x \in \Omega$ means the complex permittivity.

Now, one can eliminate the magnetic field from the above equations by dividing the first equation in (1.3) by $\mu$ and taking the curl to obtain the following system of equations for $\mathbf{E}$ :

$$
\nabla \times\left(\mu^{-1} \nabla \times \mathbf{E}\right)-\omega^{2} \varepsilon \mathbf{E}=0 \quad \text { in } \Omega .
$$

Having found the electric field $\mathbf{E}$, we then obtain the magnetic field $\mathbf{H}$ through the formula:

$$
\mathbf{H}=\frac{1}{i \omega \mu} \nabla \times \mathbf{E} .
$$

It is well known that to get particular non-trivial solutions to (1.4), one can prescribe non-trivial boundary conditions for $\mathbf{E} \times \nu$, on the boundary of the domain $\Omega$ with $\nu$ means the outward unit normal to $\Omega$.

Let $\mu_{0}>0, \varepsilon_{0}^{r e}>0$ and $\sigma_{0}$ denote the permeability, the (real) permittivity, and the conductivity of the background medium, by means of $\varepsilon_{0}=\varepsilon_{0}^{r e}+i \frac{\sigma_{0}}{\omega}$ denotes the background complex permittivity. Also, we denote by $\mu_{1}>0, \varepsilon_{1}^{r e}>0$ and $\sigma_{1}$ respectively the permeability, the (real) permittivity, and the conductivity of the inhomogeneity with $\varepsilon_{1}=\varepsilon_{1}^{r e}+i \frac{\sigma_{1}}{\omega}$ means the associated complex permittivity. For simplicity, we shall assume that all these parameters are constants and that $\sigma_{0}<<\omega \varepsilon_{0}^{r e}, \sigma_{1}<<\omega \varepsilon_{1}^{r e}$. In that case $\varepsilon_{0} \approx \varepsilon_{0}^{r e}$ and $\varepsilon_{1} \approx \varepsilon_{1}^{r e}$. We denote by $k=\omega \sqrt{\varepsilon \mu}>0$ the wave number, where $\omega>0$ is a given frequency.

Using this notation we introduce the piecewise constant magnetic permeability:

$$
\mu_{\delta}(x)= \begin{cases}\mu_{0}, & x \in \Omega \backslash \bar{D}, \\ \mu_{1}, & x \in D .\end{cases}
$$

If we allow the degenerate case $\delta=0$, then the function $\mu_{0}(x)$ equals the constant $\mu_{0}$. The piecewise constant electric permittivity, $\varepsilon_{\delta}$ is defined analogously. The electric field in the presence of the inhomogeneity, is denoted $\mathbf{E}_{\delta}$. It is the solution to the following Maxwell's equations:

$$
\left\{\begin{array}{l}
\nabla \times\left(\frac{1}{\mu_{\delta}} \nabla \times \mathbf{E}_{\delta}\right)-\omega^{2} \varepsilon_{\delta} \mathbf{E}_{\delta}=0 \quad \text { in } \Omega, \\
\mathbf{E}_{\delta} \times \nu=\mathbf{g}, \quad \text { on } \partial \Omega,
\end{array}\right.
$$


where $\mathbf{g}$ is a tangential field on the boundary $\partial \Omega$ that furthermore belongs to an adequate Sobolev space.

Considering (1.5), we find that the magnetic field $\mathbf{H}_{\delta}$ solves:

$$
\left\{\begin{array}{l}
\nabla \times\left(\frac{1}{\varepsilon_{\delta}} \nabla \times \mathbf{H}_{\delta}\right)-\omega^{2} \mu_{\delta} \mathbf{H}_{\delta}=0 \quad \text { in } \Omega, \\
\frac{1}{\varepsilon_{\delta}}\left(\nabla \times \mathbf{H}_{\delta}\right) \times \nu=\mathbf{g}, \quad \text { on } \partial \Omega .
\end{array}\right.
$$

The equations in (1.7) may alternatively be formulated as follows:

$$
\begin{aligned}
& \nabla \times\left(\nabla \times \mathbf{E}_{\delta}\right)=\omega^{2} \mu_{0} \varepsilon_{0} \mathbf{E}_{\delta}, \quad \nabla \cdot\left(\mathbf{E}_{\delta}\right)=0 \quad \text { in } \Omega \backslash \bar{D} \\
& \nabla \times\left(\nabla \times \mathbf{E}_{\boldsymbol{\delta}}\right)=\omega^{2} \mu_{1} \varepsilon_{1} \mathbf{E}_{\boldsymbol{\delta}}, \quad \nabla \cdot\left(\mathbf{E}_{\delta}\right)=0 \quad \text { in } D, \\
& \quad \mathbf{E}_{\delta} \times \nu \text { is continuous across } \partial D, \\
& \left.\frac{1}{\mu_{0}}\left(\nabla \times \mathbf{E}_{\delta}\right)\right|_{+} \times \nu-\left.\frac{1}{\mu_{1}}\left(\nabla \times \mathbf{E}_{\delta}\right)\right|_{-} \times \nu=0 \quad \text { on } \partial D, \\
& \left.\varepsilon_{0} \mathbf{E}_{\delta}\right|_{+} \cdot \nu-\left.\varepsilon_{1} \mathbf{E}_{\delta}\right|_{-} \cdot \nu=0 \quad \text { on } \partial D, \\
& \quad \mathbf{E}_{\delta} \times \nu=\mathbf{g} \quad \text { on } \partial \Omega .
\end{aligned}
$$

Here $\nu$ denotes the outward unit normal to $\partial D$ (and to $\partial \Omega$ ); superscript + and - indicate the limiting values as we approach $\partial D$ from outside $D$, and from inside $D$, respectively.

The electric field, $\mathbf{E}_{0}$, in the absence of inhomogeneity, satisfies:

$$
\begin{gathered}
\nabla \times\left(\nabla \times \mathbf{E}_{0}\right)=\omega^{2} \mu_{0} \varepsilon_{0} \mathbf{E}_{0}, \quad \nabla \cdot\left(\mathbf{E}_{0}\right)=0 \quad \text { in } \Omega, \\
\mathbf{E}_{0} \times \nu=\mathbf{g} \quad \text { on } \partial \Omega .
\end{gathered}
$$

We assume that

$$
" \omega \text { is not a resonant frequency for the problem (1.15)-(1.16)" }
$$

and so by assumption there exists a unique solution $\left(\mathbf{E}_{0}, \mathbf{H}_{0}\right) \in[H(\operatorname{curl}, \Omega)]^{2}=$ $\left\{\mathbf{v} \in L^{2}(\Omega)^{3} \text {, curl } \mathbf{v} \in L^{2}(\Omega)^{3}\right\}^{2}$ of $(1.3)$ if $\delta=0$.

It has been shown in [4] that assumption (1.17) insures also well-posedness for the $\delta$-dependent case for $\delta$ sufficiently small, that are problems (1.3) and (1.9)-(1.14). The main achievement of this paper is a rigorous derivation, based on layer potential techniques, of complete asymptotic expansions of $\mathbf{E}_{\delta} \times \nu, \mathbf{H}_{\delta} \times \nu, \nabla \times \mathbf{E}_{\delta} \times \nu$ as $\delta \rightarrow 0$.

These formulas generalize those by Vogelius and Volkov [20, 14], where only solutions with a transverse electric and a transverse magnetic symmetries were considered. In $[2,4,3]$, Ammari et al. have also derived formulas for the perturbations in boundary integrals of solutions to the time-harmonic Maxwell equations. Thanks to electromagnetic potentials, we decide to go more in this line of work, and to advance the 
generalization of related previous results. For example, in [2] we have presented only formal derivations of asymptotic expansions of the solutions to Maxwell equations. Moreover, these results are based on Lippman-Schwinger integral representation formula which provides us only with asymptotic formulae at lower orders. Conversely, in the present paper we use layer technique methods based on formulations introduced before in $[2,18,19]$ and used in several works of Buffa et al. $[6,7]$ and Costabel et al.[10, 11, 12, 13]. Additionally, we refer the readers to the works $[17,16]$. These

authors have used different techniques from harmonic analysis to develop a theory of boundary integral equations for the time-harmonic Maxwell equations on Lipschitz domains. For a same approach, we find the potential work of Ammari and Kang in [1] where a complete asymptotic expansion of solutions to the Helmholtz equation is obtained. Moreover, the general approach developed in this paper could be carried out to obtain more precise asymptotic formulas for the full Maxwell's equations than those derived in [3] and [5]. The proofs of our asymptotic expansions are radically different from the ones found in above cited references. They are based on layer potential techniques and a new decomposition formula of the solution to the Maxwell equation. This decomposition formula generalizes that introduced by Ammari and Kang [1] for the Helmholtz equation, and by Kang and Seo in [15] for steady-state voltage potentials.

We note that our formulas will find important applications for developing effective algorithms for reconstructing small dielectric inhomogeneities from electromagnetic boundary measurements which can be applied for medical imagining, breast cancer, and land mines.

The outline of this paper is as follows. Section 2 is devoted to the introduction of some notations and preliminaries regarding layer potentials. In section 3 , we give different representations of solution of the underlined problem. In section 4, after giving an estimate to the difference $\mathbf{E}_{\delta}-\mathbf{E}_{0}$, we provide a rigorous derivation of complete asymptotic expansions of tangential traces of both electric and magnetic fields. Based on electromagnetic potentials, we provide also complete asymptotic expansions of the electric field $\mathbf{E}_{\delta}(x)$ for $x \in \Omega$. For $d=2$, we give a rigorous asymptotic expansion of $\mathbf{E}_{\delta}(x)$ at order 2 which generalize the one obtained by matched expansions in [2] for the time-harmonic Maxwell's equations with TE symmetry.

\section{Preliminary results}

We will develop a boundary integral formulation for solving time-harmonic Maxwell equations. The theory of layer potentials has been developed in relation to the boundary value problems. In this investigation we will recall and we will use both electric and magnetic layer potentials for proving our complete asymptotic expansion of solution when the shape parameter $\delta \rightarrow 0$. 
Firstly, we present some results on the characterization of traces associated to Sobolev spaces of interest for Maxwell's equations.

\subsection{Functional spaces}

In this paper, we denote by bold letters the functional spaces for the vector fields in $\mathbb{R}^{d}$. Thus, we denote by $\mathcal{D}(\Omega)$ the space of the $3 D$ vector fields with each component belonging to $C_{0}^{\infty}(\Omega)$ and by $\mathcal{D}^{\prime}(\Omega)$ the corresponding dual space. The duality is denoted by $<\cdot, \cdot>_{\mathcal{D}}$. Moreover, $H^{s}(\Omega)$ denotes the usual Sobolev space on $\Omega$ and $\mathbf{H}^{s}(\Omega)$ denotes $\left(H^{s}(\Omega)\right)^{d}$ and $\mathbf{L}^{2}(\Omega)$ denotes $\left(L^{2}(\Omega)\right)^{d}$. As usual for Maxwell equations, we recall that if the domain $\Omega$ is regular, all the definitions here below make sense and are correct (see $[6,8,21])$. Let us set:

$$
\begin{aligned}
H(\operatorname{curl}, \Omega) & :=\left\{\mathbf{v} \in \mathbf{L}^{2}(\Omega) ; \operatorname{curl} \mathbf{v} \in \mathbf{L}^{2}(\Omega)\right\}, \quad\|\cdot\|_{H(\operatorname{curl}, \Omega)} \text { the graph norm, } \\
H(\operatorname{div}, \Omega) & :=\left\{\mathbf{v} \in \mathbf{L}^{2}(\Omega) ; \operatorname{div} \mathbf{v} \in \mathbf{L}^{2}(\Omega)\right\}, \quad\|\cdot\|_{H(\operatorname{div}, \Omega)} \text { the graph norm, } \\
& :=\{\mathbf{v} \times \nu: \mathbf{v} \in H(\operatorname{curl}, \Omega)\}, \\
\mathbf{H}^{s}(\partial \Omega) & :=\left(H^{s}(\partial \Omega)\right)^{d} \quad \text { for } s>0, \text { and } \mathbf{H}^{0}(\partial \Omega):=\mathbf{L}^{2}(\partial \Omega) .
\end{aligned}
$$

\subsection{Layer potentials for the Helmholtz equation}

Let us first review some well-known properties of the layer potentials for the Helmholtz equation and recall some useful identities.

Let $D$ be a bounded domain in $\mathbb{R}^{d}, d=2,3$. We assume that $\partial D$ is Lipschitz. Let $\Phi_{k}(x)$ be the fundamental solution for $\Delta+k^{2}$, that is for $x \neq 0$ :

$$
\Phi_{k}(x)= \begin{cases}-\frac{i}{4} H_{0}^{1}(k|x-y|), & d=2, \\ -\frac{e^{i k|x-y|}}{4 \pi|x-y|}, & d=3\end{cases}
$$

where $H_{0}^{1}$ is the Hankel function of the first kind of order 0. Similarly, the fundamental solution for the Laplacian is for $x \neq 0$ :

$$
\Phi_{0}(x)= \begin{cases}\frac{1}{2 \pi} \log |x-y|, & d=2, \\ -\frac{1}{4 \pi|x-y|}, & d=3 .\end{cases}
$$

The single and double layer potentials of the density function $\varphi$ on $D$ is defined 
by:

$$
\begin{aligned}
\mathcal{S}_{D}^{k} \varphi(x) & :=\int_{\partial D} \Phi_{k}(x-y) \varphi(y) d \sigma(y), \quad x \in \mathbb{R}^{d}, \\
\mathcal{D}_{D}^{k} \varphi(x) & :=\int_{\partial D} \frac{\partial}{\partial \nu_{y}} \Phi_{k}(x-y) \varphi(y) d \sigma(y), \quad x \in \mathbb{R}^{d} \backslash \partial D .
\end{aligned}
$$

\subsection{Electromagnetic potentials}

We use some well known results about traces of vector fields and integral representations of time-harmonic electromagnetic fields. Details can be found in $[6,7,8,10,18]$.

For a vector function $\mathbf{u} \in\left(C^{\infty}(\bar{\Omega})\right)^{d}$ and a scalar function $v \in C^{\infty}(\bar{\Omega})$ we define the traces :

$$
\begin{aligned}
\gamma(\mathbf{u}) & =\left.\mathbf{u}\right|_{\partial \Omega}, \\
\gamma_{D i r}(\mathbf{u}) & =\left.(\nu \times \mathbf{u})\right|_{\partial \Omega} \quad \text { Dirichlet conditions } \\
\gamma_{N e u}(\mathbf{u}) & =\left.(\nu \times \operatorname{curlu})\right|_{\partial \Omega} \quad \text { Neumann conditions. }
\end{aligned}
$$

It is well known that for $s>0$, the traces

$$
\begin{aligned}
\gamma: \mathbf{H}^{s+1 / 2}(\Omega) & \rightarrow \mathbf{H}^{s}(\partial \Omega), \\
\gamma_{\text {Dir }}: \mathbf{H}^{s+1 / 2}(\Omega) & \rightarrow \mathbf{H}_{*}^{s}(\partial \Omega)
\end{aligned}
$$

are continuous.

According to [18], we can define some useful surface differential operators such that the tangential gradient denoted by $\nabla_{\partial \Omega}$, the surface divergence denoted by $\operatorname{div}_{\partial \Omega}$, and the tangential vector curl denoted by $\operatorname{curl}_{\partial \Omega}$. Now, using previous definitions we can define the Hilbert space:

$$
\left.T H_{\operatorname{div}}^{-1 / 2}(\partial \Omega):=\left\{\mathbf{v} \in \mathbf{H}^{-1 / 2}(\partial \Omega) ; \mathbf{v} \cdot \nu=0, \operatorname{div} \partial \Omega \mathbf{v} \in H^{-1 / 2}(\partial \Omega)\right)\right\}
$$

endowed with the norm:

$$
\|\cdot\|_{T H_{\operatorname{div}}^{-1 / 2}(\partial \Omega)}=\|\cdot\|_{\mathbf{H}^{-1 / 2}(\partial \Omega)}+\|\operatorname{div} \cdot\|_{H^{-1 / 2}(\partial \Omega)} \cdot
$$

Remark 2.1 The single layer potential defined in (2.3) is applied also to tangent vectors and, since it is never misleading, we shall use the same notations.

We define the electric potential $\mathcal{V}_{D}^{k}$ generated by $\mathbf{j} \in T H_{\operatorname{div}}^{-1 / 2}(\partial D)$ by

$$
\mathcal{V}_{D}^{k} \mathbf{j}=k \mathcal{S}_{D}^{k} \mathbf{j}+1 / k \nabla \mathcal{S}_{D}^{k} \operatorname{div}_{\partial \Omega \mathbf{j}}
$$


Analogously, we define the magnetic potential $\mathcal{W}_{D}^{k}$ generated by $\mathbf{m} \in T H_{\operatorname{div}}^{-1 / 2}(\partial D)$ by

$$
\mathcal{W}_{D}^{k} \mathbf{m}=\operatorname{curl} \mathcal{S}_{D}^{k} \mathbf{m} \text {. }
$$

The following results can be found in $[7,10,12]$, for example.

Lemma $2.2([7])$ Let $B$ be a bounded domain with a smooth boundary B. Then, the potentials $\mathcal{V}_{B}^{k}$ and $\mathcal{W}_{B}^{k}$ defined by (2.5) and (2.6) respectively are continuous from $T H_{\text {div }}^{-1 / 2}(\partial B)$ to $H($ curl $; B)$. Moreover, for $\mathbf{j} \in T H_{\text {div }}^{-1 / 2}(\partial B)$ we have, for all $x \in R^{d} \backslash \partial B$ :

$$
\begin{array}{r}
\left(\text { curlcurl }-k^{2} \mathbf{I}\right) \mathcal{V}_{B}^{k} \mathbf{j}(x)=0 \\
\left(\text { curlcurl }-k^{2} \mathbf{I}\right) \mathcal{W}_{B}^{k} \mathbf{m}(x)=0
\end{array}
$$

where $\mathbf{I}$ is the identity operator.

Moreover, the following jump conditions hold, on $\partial D$, as given in [7]:

$$
\begin{aligned}
& k \gamma_{\text {Dir }}\left(\mathcal{V}_{D}^{k}\right)=\gamma_{\text {Neu }}\left(\mathcal{W}_{B}^{k}\right), \\
& k \gamma_{\text {Dir }}\left(\mathcal{W}_{D}^{k}\right)=\gamma_{\text {Neu }}\left(\mathcal{V}_{B}^{k}\right)
\end{aligned}
$$

\section{Representation of solutions}

Our aim in this section is to give a representation of the solution of (1.9)-(1.14) through boundary layer techniques. This representation generalizes those found in [1] for Helmholtz Equation and in [15] for the harmonic equation. Our first step in this investigation, is to extend the well-known Stratton-Chu integral representation to electromagnetic fields $(\mathbf{E}, \mathbf{H}) \in(H(\operatorname{curl}, \Omega))^{2}$.

Recall the well known representation (see for example $[7,11]$ ).

Lemma 3.1 (Stratton-Chu) Suppose that $\mathcal{O}$ is a $C^{2}$ regular bounded domain in $R^{3}$. Let $\nu$ be the unit outward normal to $\partial \mathcal{O}$, and let $(\mathbf{E}, \mathbf{H}) \in C^{1}(\overline{\mathcal{O}}) \times C^{1}(\overline{\mathcal{O}})$ solution of the time-harmonic Maxwell equations

$$
\left\{\begin{array}{l}
\nabla \times \mathbf{E}-i k \mathbf{H}=0, \quad \text { in } \mathcal{O}, \\
\nabla \times \mathbf{H}+i k \varepsilon \mathbf{E}=0, \quad \text { in } \mathcal{O} \\
\text { B.C, on } \partial \mathcal{O},
\end{array}\right.
$$

where $k=\omega \sqrt{\mu \varepsilon}>0$ is the associated wave number. Then for all $x \in \mathcal{O}$, we have:

$$
\mathbf{E}(x)=-\nabla \times \int_{\partial \mathcal{O}} \nu(y) \times \mathbf{E}(y) \Phi(x-y) d \sigma(y)+\nabla \int_{\partial \mathcal{O}} \nu(y) \cdot \mathbf{E}(y) \Phi(x-y) d \sigma(y)
$$




$$
\begin{gathered}
-i k \int_{\partial \mathcal{O}} \nu(y) \times \mathbf{H}(y) \Phi(x-y) d \sigma(y)+i k \int_{\mathcal{O}}[\nabla \times \mathbf{H}(y)+i k \mathbf{E}(y)] \Phi(x-y) d y \\
-\nabla \int_{\mathcal{O}} \nabla \cdot \mathbf{E}(y) \Phi(x-y) d y+\nabla \times \int_{\mathcal{O}}[\nabla \times \mathbf{E}(y)-i k \mathbf{H}(y)] \Phi(x-y) d y
\end{gathered}
$$

with $\Phi$ the fundamental solution of the Helmholtz equation.

According to [11], representation $(3.2)$ holds also for $(\mathbf{E}, \mathbf{H}) \in H(\operatorname{curl}, \Omega) \times H(\operatorname{curl}, \Omega)$, where the boundary values are understood in the sense of weak tangential and normal traces in $H^{-1 / 2}(\partial \mathcal{O})$.

Now, one can use Lemma 3.1 to get the first integral representation of the solution to problem (1.9)-(1.14).

Proposition 3.2 Suppose that $D$ is a domain compactly contained in $\Omega$ with a connected Lipschitz boundary and wave number $k$. Suppose that we have condition (1.17). Let $(\mathbf{E}, \mathbf{H})$ be the solution of (1.3) and the Cauchy data $\mathbf{j}=[\nu \times \mathbf{E}]_{\partial D}$, and $\mathbf{m}=[\nu \times \mathbf{E}]_{\partial D}$. Then, if we set $\mathbf{j}_{0}=\nu \times\left.\mathbf{H}_{0}\right|_{\partial \Omega}$, and $\mathbf{m}_{0}=\nu \times\left.\mathbf{E}_{0}\right|_{\partial \Omega}$, we immediately get:

$$
\begin{aligned}
\mathbf{E}(x)=- & \int_{\partial D} \Phi_{k_{0}}(x, y) \mathbf{j}(y) d \sigma(y)-\frac{1}{\mu_{0} \varepsilon_{0} \omega^{2}} \nabla \int_{\partial D} \Phi_{k_{0}}(x, y)_{\partial D} \mathbf{j}(y) d \sigma(y) \\
& -\nabla \times \int_{\partial \Omega} \Phi_{k_{0}}(x, y) \mathbf{m}_{0}(y) d \sigma(y)+\int_{\partial \Omega} \Phi_{k_{0}}(x, y) \mathbf{j}_{0}(y) d \sigma(y) \\
& +\frac{1}{\mu_{0} \varepsilon_{0} \omega^{2}} \nabla \int_{\partial \Omega} \Phi_{k_{0}}(x, y)_{\partial \Omega} \mathbf{j}_{0}(y) d \sigma(y), \quad \text { for } x \in \Omega \backslash \bar{D}
\end{aligned}
$$

and,

$$
\mathbf{E}(x)=-\int_{\partial D} \Phi_{k}(x, y) \mathbf{j}(y) d \sigma(y)-\frac{1}{\mu \varepsilon \omega^{2}} \nabla \int_{\partial D} \Phi_{k}(x, y)_{\partial D} \mathbf{j}(y) d \sigma(y), \quad \text { for } x \in D,
$$

where $\Phi_{k}$ is given by (2.1).

Proof. Let $\mathbf{E}(x)$ be the solution of the problem (1.9)-(1.14). Then, with the Stratton-Chu representation formula (3.2), we may represent the solution $\mathbf{E}(x)$ in $\Omega$ as follows:

$$
\begin{aligned}
& \tilde{\mathbf{E}}(x)=-\nabla \times \int_{\partial \Omega \cup \partial D} \nu(y) \times \tilde{\mathbf{E}}(y) \Phi_{\tilde{k}}(x-y) d \sigma(y)+\nabla \int_{\partial \Omega \cup \partial D} \nu(y) \cdot \tilde{\mathbf{E}}(y) \Phi_{\tilde{k}}(x-y) d \sigma(y) \\
& -i \tilde{k} \int_{\partial \Omega \cup \partial D} \nu(y) \times \tilde{\mathbf{H}}(y) \Phi_{\tilde{k}}(x-y) d \sigma(y)+i \tilde{k} \int_{(\Omega \backslash \bar{D}) \cup D}(\nabla \times \tilde{\mathbf{H}}(y)+i \tilde{k} \tilde{\mathbf{E}}(y)) \Phi_{\tilde{k}}(x-y) d y
\end{aligned}
$$


$-\nabla \int_{(\Omega \backslash \bar{D}) \cup D} \nabla \cdot \tilde{\mathbf{E}}(y) \Phi_{\tilde{k}}(x-y) d y+\nabla \times \int_{(\Omega \backslash \bar{D}) \cup D}(\nabla \times \tilde{\mathbf{E}}(y)-i \tilde{k} \tilde{\mathbf{H}}(y)) \Phi_{\tilde{k}}(x-y) d y$ where,

$$
\tilde{\mathbf{E}}=\left\{\begin{array}{ll}
\mathbf{E}_{0}, & \text { in } \Omega \backslash \bar{D}, \\
\mathbf{E}, & \text { in } D,
\end{array}, \quad \tilde{\mathbf{H}}= \begin{cases}\mathbf{H}_{0}, & \text { in } \Omega \backslash \bar{D}, \\
\mathbf{H}, & \text { in } D,\end{cases}\right.
$$

and $\tilde{k}=\left\{\begin{array}{ll}k_{0}, & \text { in } \Omega \backslash \bar{D}, \\ k, & \text { in } D .\end{array}\right.$.

For more details about the proof of last integral relation, we refer the readers to [18] and [9].

Now, inserting relations found in (1.3) and (1.9)-(1.10) into last relation (3.2), immediately volume integrals vanish. Thus, to get the desired results we insert appropriate boundary conditions.

Based on the trace operators and the results found in Proposition 3.2, it is easy to prove the following transmission conditions.

Lemma 3.3 Suppose that we have all hypothesis of Proposition 3.2. Let $\mathbf{f}=\mathbf{H}_{0} \times$ $\left.\nu\right|_{\partial \Omega}$, and $\mathbf{g}=\mathbf{E}_{0} \times\left.\nu\right|_{\partial \Omega}$. Then, if $\mathbf{j} \in T H_{\text {div }}^{-1 / 2}(\partial D)$ solves (3.3)-(3.4) for $\mathbf{E}$ solution of (1.9)-(1.14), the following boundary conditions hold:

$$
\begin{gathered}
\mathbf{g}(x)=\left(\mathcal{V}_{\Omega}^{k_{0}} \mathbf{f}(x)+\mathcal{W}_{\Omega}^{k_{0}} \mathbf{g}(x)\right) \times \nu(x)-\mathcal{V}_{D}^{k_{0}} \mathbf{j}(x) \times \nu(x), \quad x \in \partial \Omega \\
-\mathcal{V}_{D}^{k} \mathbf{j}(x) \times \nu(x)=\left(\mathcal{V}_{\Omega}^{k_{0}} \mathbf{f}(x)+\mathcal{W}_{\Omega}^{k_{0}} \mathbf{g}(x)\right) \times \nu(x)-\mathcal{V}_{D}^{k_{0}} \mathbf{j}(x) \times \nu(x), \quad x \in \partial D \\
-\varepsilon_{1} \mathcal{V}_{D}^{k} \mathbf{j}(x) \cdot \nu(x)=\varepsilon_{0}\left(\mathcal{V}_{\Omega}^{k_{0}} \mathbf{f}(x)+\mathcal{W}_{\Omega}^{k_{0}} \mathbf{g}(x)\right) \cdot \nu(x)-\varepsilon_{0} \mathcal{V}_{D}^{k_{0}} \mathbf{j}(x) \cdot \nu(x), \quad x \in \partial D \\
-\frac{1}{\mu_{1}} \mathcal{V}_{D}^{k} \mathbf{j}(x) \times \nu(x)=\frac{1}{\mu_{0}}\left(\mathcal{V}_{\Omega}^{k_{0}} \mathbf{f}(x)+\mathcal{W}_{\Omega}^{k_{0}} \mathbf{g}(x)\right) \times \nu(x) \\
-\frac{1}{\mu_{0}} \mathcal{V}_{D}^{k_{0}} \mathbf{j}(x) \times \nu(x), \quad x \in \partial D
\end{gathered}
$$

The potential operators $\mathcal{V}_{D}^{k}$ and $\mathcal{W}_{D}^{k}$ are defined by (2.5) and (2.6) respectively.

The following result gives the counterverse of that proved in Proposition 3.2.

Theorem 3.4 Suppose that we have all hypothesis of Proposition 3.2. Let $\mathbf{j}_{0}=$ $\nu \times\left.\mathbf{H}_{0}\right|_{\partial \Omega}$, and $\mathbf{m}_{0}=\nu \times\left.\mathbf{E}_{0}\right|_{\partial \Omega}$. Then, if $\mathbf{j} \in T H_{\text {div }}^{-1 / 2}(\partial D)$ solves (3.3)-(3.4), the vector valued function $\mathbf{E}$ given by:

$$
\mathbf{E}(x)=-\mathcal{V}_{\Omega}^{k_{0}} \mathbf{j}_{0}(x)-\mathcal{W}_{\Omega}^{k_{0}} \mathbf{m}_{0}(x)-\mathcal{V}_{D}^{k_{0}} \mathbf{j}(x), \quad \text { for } x \in \Omega \backslash \bar{D},
$$


and,

$$
\mathbf{E}(x)=-\mathcal{V}_{D}^{k} \mathbf{j}(x) \quad \text { for } x \in D
$$

solves the problem (1.9)-(1.14).

Proof. According to (2.7)-(2.8) and Remark 2.1, the potentials $\mathcal{V}_{\Omega}^{k_{0}}$ and $\mathcal{W}_{\Omega}^{k_{0}}$ satisfy the Maxwell equations. Then, in each case (in $\Omega \backslash \bar{D}$ or in $D$ ) the integral representations of $\mathbf{E}$ satisfy the Maxwell equations (1.9)-(1.10). It remains to prove that the boundary conditions (1.11)-(1.14) are satisfied if the density $\mathbf{j}$ satisfies a specific equations. But, by simple computations in (3.10) and in (3.11), one can deduce easily the desired boundary conditions from relations (3.6)-(3.9) given by Lemma 3.3. Our result is then proved.

To explain more our principal results in this paper, we may exploit Proposition 3.2 to expand solution of (1.9)-(1.14) into different terms. This technique was used firstly by Kang and Seo in [15], and later was more developed by Ammari and Kang in [1] for Helmholtz problem. Our strategy, now, is to go more with this method by providing a generalization to time-harmonic Maxwell equations. Then, the following main representation holds.

Theorem 3.5 Suppose that we have all hypothesis of Proposition 3.2. Let $\mathbf{E}$ be the solution of (1.9)-(1.14) with $\mathbf{f}=-\gamma_{N e u}\left(\left.\mathbf{E}\right|_{\partial \Omega}\right)$ and $\mathbf{g}=-\left.\gamma_{\text {Dir }}(\mathbf{E})\right|_{\partial \Omega}$. Define

$$
\mathcal{H}(x):=\mathcal{V}_{\Omega}^{k_{0}} \mathbf{f}(x)+\mathcal{W}_{\Omega}^{k_{0}} \mathbf{g}(x), \quad x \in \mathbb{R}^{d} \backslash \partial \Omega,
$$

and let $(\mathbf{j}, \mathbf{m}) \in T H_{d i v}^{-1 / 2}(\partial D) \times T H_{d i v}^{-1 / 2}(\partial D)$ be the unique solution of

$$
\left\{\begin{array}{l}
\mathcal{V}_{D}^{k} \mathbf{j} \times \nu-\mathcal{V}_{D}^{k_{0}} \mathbf{m} \times \nu=\mathcal{H} \times \nu \quad \text { on } \partial D \\
\frac{1}{\mu_{1}} \gamma_{N e u}\left(\mathcal{V}_{D}^{k} \mathbf{j}\right)-\frac{1}{\mu_{0}} \gamma_{N e u}\left(\mathcal{V}_{D}^{k_{0}} \mathbf{m}\right)=\frac{1}{\mu_{0}} \gamma_{\text {Neu }}(\mathcal{H}) \quad \text { on } \partial D \\
\varepsilon_{1} \mathcal{V}_{D}^{k} \mathbf{j} \cdot \nu-\varepsilon_{0} \mathcal{V}_{D}^{k_{0}} \mathbf{m} \cdot \nu=\varepsilon_{0} \mathcal{H} \cdot \nu \quad \text { on } \partial D
\end{array}\right.
$$

Then $\mathbf{E}$ can be represented by:

$$
\mathbf{E}(x)=\left\{\begin{array}{l}
\mathcal{V}_{D}^{k_{0}} \mathbf{m}(x)+\mathcal{H}(x), \quad x \in \Omega \backslash \bar{D} \\
\mathcal{V}_{D}^{k} \mathbf{j}(x) \quad x \in D .
\end{array}\right.
$$

Moreover, $\forall n \in N$, there exists a constant $C_{n}=C(n, \Omega$, dist $(D, \partial \Omega))$ independent of $|D|$ such that

$$
\|\mathcal{H}\|_{\mathcal{C}^{n}(\bar{D})} \leq C_{n} \quad\|\mathbf{g}\|_{T H_{d i v}^{-1 / 2}(\partial \Omega)}
$$

Proof. From Lemma 2.2, we know that the electric potential operators $\mathcal{V}_{D}^{k_{0}} \mathbf{m}(x)$ and the magnetic potential operator $\mathcal{W}_{D}^{k_{0}} \mathbf{m}(x)$ satisfy the Maxwell equations (2.7), 
(2.8) respectively. Then, the vector valued function $\mathcal{H}$ defined by (3.12) satisfies the same Maxwell equations. Moreover, the fact that the densities $\mathbf{j}$ and $\mathbf{m}$ solve uniquely the system of equations (3.13), we conclude that the field $\mathbf{E}$ given by (3.14) solves the equations (1.9)-(1.14). From Lemma 2.2, we know that the electric potential operators $\mathcal{V}_{D}^{k_{0}} \mathbf{m}(x)$ and the magnetic potential operator $\mathcal{W}_{D}^{k_{0}} \mathbf{m}(x)$ satisfy the Maxwell equations (2.7), (2.8) respectively. Conversely, to justify representation (3.14), we may use Theorem 3.4 which guarantees that representation (3.14) solves the problem (1.9)-(1.14). So that by uniqueness condition, the solution $\mathbf{E}$ satisfies (3.14).

The representation formula (3.15) was proved in [15] for the steady sate problem, and in [1] for the Helmholtz problem and it is easily seen that the same proof works for the full Maxwell equations.

Based on definition (3.12), it is not hard to find a positive constant $C=C(n, \operatorname{dist}(D, \partial \Omega))$ such that:

$$
\|\mathcal{H}\|_{C^{n}(\bar{D})} \leq C\left(\|f\|_{T H_{\mathrm{div}}^{-1 / 2}(\partial \Omega)}+\|g\|_{T H_{\mathrm{div}}^{-1 / 2}(\partial \Omega)}\right),
$$

where $f=\operatorname{curl} \mathbf{E} \times\left.\nu\right|_{\partial \Omega}, g=\mathbf{E} \times\left.\nu\right|_{\partial \Omega}$ and $n$ a positive integer.

To obtain our main estimation, we may use the natural weak formulation of the electric problem that, for $\mathbf{E} \in H(\operatorname{curl}, \Omega)$ :

$$
a(\mathbf{E}, \mathbf{v})=l(\mathbf{v}) \quad \text { for all } \mathbf{v} \in H(\operatorname{curl}, \Omega),
$$

where the conjugate-linear functional $l(\mathbf{v})=\int_{\partial \Omega} \mathbf{g} \cdot \overline{\mathbf{v}} d \sigma$ is to be interpreted as the duality pairing on $\partial \Omega$ between the appropriate spaces of distributions and test functions. But, $a(\mathbf{E}, \mathbf{v})$ denotes the sesquilinear form:

$$
a(\mathbf{E}, \mathbf{v})=\int_{\Omega} \frac{1}{\mu} \nabla \times \mathbf{E} \cdot \overline{\nabla \times \mathbf{v}} d x-\omega^{2} \int_{\Omega} \varepsilon \mathbf{E} \cdot \overline{\mathbf{v}} d x .
$$

It is well known that if $\omega^{2}$ is not an eigenfrequency of the Maxwell problem (1.3), the solution $\mathbf{E}$ is the unique solution to weak formulation (3.17) such that:

$$
\|\mathbf{E}\|_{H(\operatorname{curl} ; \Omega)} \leq C\|\mathbf{g}\|_{T H_{\text {div }}^{-1 / 2}(\partial \Omega)},
$$

where the positive constant $C$ depends only on the medium parameters $\mu, \varepsilon, \mu_{0}$ and $\varepsilon_{0}$.

Let $\mathbf{v} \in T H_{\operatorname{div}}^{-1 / 2}(\partial \Omega)$. For such a datum $\mathbf{v}$, let us consider $\tilde{\mathbf{v}} \in H(\operatorname{curl} ; \Omega)$ such that:

$$
\left\{\begin{array}{l}
\mathbf{v}=\tilde{\mathbf{v}} \times \nu, \quad \text { on } \partial \Omega \\
\|\tilde{\mathbf{v}}\|_{H(\operatorname{curl} ; \Omega)} \leq C_{1}\|\mathbf{v}\|_{T H_{\operatorname{div}}^{-1 / 2}(\partial \Omega)}
\end{array}\right.
$$

where $C_{1}=C_{1}(\Omega)$ is a positive constant depending only on $\Omega$. Note that affirmation (3.19) can be deduced from $[3,5]$. 
Analogously to formulation (3.17), we may write a formulation for the magnetic field $\mathbf{H}$ as follows:

$$
\int_{\partial \Omega} \mathbf{f} \cdot \overline{\mathbf{v}} d \sigma=\int_{\Omega} \frac{1}{\varepsilon} \nabla \times \psi \mathbf{H} \cdot \overline{\nabla \times \mathbf{v}} d x-\omega^{2} \int_{\Omega} \mu \psi \mathbf{H} \cdot \overline{\mathbf{v}} d x
$$

where $\psi$ is a smooth function, with $\psi=1$ in a neighborhood of $\partial \Omega$ and $\psi=0$ in $D$. Now, applying Cauchy-Schawrtz inequality to relation (3.20), we immediately get:

$$
\left|\int_{\partial \Omega} \mathbf{f} \cdot \overline{\mathbf{v}} d \sigma\right| \leq C\left(\|\nabla \times \mathbf{H}\|_{L^{2}(\Omega \backslash \bar{D})}\|+\| \mathbf{H} \|_{L^{2}(\Omega \backslash \bar{D})}\right)\|\tilde{\mathbf{v}}\|_{H(\operatorname{curl}, \Omega)} .
$$

By relation (1.5), the following holds:

$$
\left|\int_{\partial \Omega} \mathbf{f} \cdot \overline{\mathbf{v}} d \sigma\right| \leq C\|\mathbf{E}\|_{H(\operatorname{curl}, \Omega \backslash \bar{D})}\|\tilde{\mathbf{v}}\|_{T H_{\text {div }}^{1 / 2}(\partial \Omega)} .
$$

Thus, using (3.19) we immediately get:

$$
\left|\int_{\partial \Omega} \mathbf{f} \cdot \overline{\mathbf{v}} d \sigma\right| \leq C\|\mathbf{E}\|_{H(\operatorname{curl}, \Omega \backslash \bar{D})}\|\mathbf{v}\|_{T H_{\text {div }}^{1 / 2}(\partial \Omega)} .
$$

Since $\mathbf{v} \in T H_{\mathrm{div}}^{1 / 2}(\partial \Omega)$ is arbitrary, we obtain:

$$
\|\mathbf{f}\|_{T H_{\mathrm{div}}^{-1 / 2}(\partial \Omega)} \leq C\|\mathbf{E}\|_{H(\operatorname{curl}, \Omega \backslash \bar{D})}
$$

where $C$ depends only on $\operatorname{dist}(D, \partial \Omega)$. Note that our last inequality agrees with the result sated by Buffa et al. in [7].

Now, It follows from (3.18), that:

$$
\|\mathbf{f}\|_{T H_{\text {div }}^{-1 / 2}(\partial \Omega)} \leq C\|\mathbf{g}\|_{T H_{\text {div }}^{-1 / 2}(\partial \Omega)} .
$$

Therefore, inserting last estimation into (3.16), we obtain:

$$
\|\mathcal{H}\|_{C^{n}(\bar{D})} \leq C\|g\|_{T H_{\mathrm{div}}^{-1 / 2}(\partial \Omega)}
$$

where $C_{n}=C(n, \Omega, \operatorname{dist}(D, \partial \Omega))$. This achieves the proof.

Due to jump conditions (2.9)-(2.10), the following representation formula for the solution $\mathbf{H}$ to (1.3) holds.

Corollary 3.6 Suppose that we have all hypothesis of Theorem 3.5. Let $\mathbf{H}$ be the solution of (1.3). Let $\mathcal{H}$ be given by (3.12) and let $(\mathbf{j}, \mathbf{m}) \in L^{2}(\partial D) \times L^{2}(\partial D)$ be the unique solution of

$$
\left\{\begin{array}{l}
\gamma_{\text {Neu }}\left(\mathcal{W}_{D}^{k} \mathbf{j}\right)-\gamma_{\text {Neu }}\left(\mathcal{W}_{D}^{k_{0}} \mathbf{m}\right)=\frac{1}{i \mu_{0} \omega} \mathcal{H} \times \nu \quad \text { on } \partial D \\
\frac{1}{\mu_{1}}\left(\mathcal{W}_{D}^{k} \mathbf{j}\right) \times \nu-\frac{1}{\mu_{0}}\left(\mathcal{W}_{D}^{k_{0}} \mathbf{m}\right) \times \nu=\frac{1}{i \mu_{0}^{2} \omega} \gamma_{N e u}(\mathcal{H}) \quad \text { on } \partial D
\end{array}\right.
$$


Then $\mathbf{H}$ can be represented by:

$$
\mathbf{H}(x)=\left\{\begin{array}{l}
\frac{1}{i \mu_{0} \omega} \nabla \times \mathcal{V}_{D}^{k_{0}} \mathbf{m}(x)+\frac{1}{i \mu_{0} \omega} \nabla \times \mathcal{H}(x), \quad x \in \Omega \backslash \bar{D}, \\
\frac{1}{i \mu \omega} \nabla \times \mathcal{V}_{D}^{k} \mathbf{j}(x) \quad x \in D
\end{array}\right.
$$

To establish a more explicitly representation formula for the electromagnetic fields $(\mathbf{E}, \mathbf{H})$, we need to introduce the $3 \times 3$ matrix valued function $\mathbf{G}^{\mathbf{0}}$ that is the solution to:

$$
\begin{aligned}
& \operatorname{curl} \frac{1}{\mu_{0}} \operatorname{curl} \mathbf{G}^{\mathbf{0}}(x, y)-\omega^{2} \varepsilon_{0} \mathbf{G}^{\mathbf{0}}(x, y)=-\delta_{y} I \quad \text { in } \Omega, \\
& \mathbf{G}^{\mathbf{0}}(x, y) \times \nu=0 \quad \text { on } \partial \Omega .
\end{aligned}
$$

Here $I$ is the $3 \times 3$ identity matrix.

For Lipschitz domain $D \subset \Omega$, we define:

$$
\mathbf{G}_{D} \varphi(x):=\int_{\partial D} \mathbf{G}^{\mathbf{0}}(x, y) \varphi(y) \times \nu(y) d \sigma(y), \quad x \in \bar{\Omega},
$$

where $\varphi$ a vector valued function. Similarly, we introduce:

$$
\tilde{\mathbf{G}}_{D} \psi(x):=\int_{\partial D} \operatorname{curl} \tilde{\mathbf{G}}^{\mathbf{0}}(x, y) \psi(y) \times \nu(y) d \sigma(y), \quad x \in \bar{\Omega},
$$

where $\psi$ is a vector valued function, and $\tilde{\mathbf{G}}^{\mathbf{0}}$ is the $3 \times 3$ matrix valued function solution to:

$$
\begin{aligned}
& \operatorname{curl} \frac{1}{\varepsilon_{0}} \operatorname{curl} \tilde{\mathbf{G}}^{\mathbf{0}}(x, y)-\omega^{2} \mu_{0} \mathbf{G}^{\mathbf{0}}(x, y)=-\delta_{y} I \quad \text { in } \Omega, \\
& \frac{1}{\varepsilon_{0}} \operatorname{curl} \tilde{\mathbf{G}}^{\mathbf{0}}(x, y) \times \nu=0 \quad \text { on } \partial \Omega .
\end{aligned}
$$

On other hand, let $f_{0}=\mathbf{H}_{0} \times \nu=\frac{1}{i \mu_{0} \omega} \nabla \times \mathbf{E}_{0} \times \nu$. Then, by the divergence theorem we get:

$$
\mathbf{E}_{0}(x)=\mathcal{V}_{\Omega}^{k_{0}} \mathbf{f}_{0}(x)+\mathcal{W}_{\Omega}^{k_{0}} \mathbf{g}(x), \quad x \in \Omega .
$$

Now using above results, one can obtain the following representations.

Theorem 3.7 Suppose that $D$ is a domain compactly contained in $\Omega$ with a connected Lipschitz boundary satisfying (1.1). Let $\mathbf{E}$ be the solution of (1.9)-(1.14). Then, for the electric field we have the following representation formula:

$$
\nabla \times \mathbf{E}(x) \times \nu(x)=\nabla \times \mathbf{E}_{0}(x) \times \nu(x)+\nabla \times \mathbf{G}_{D} \mathbf{m}(x) \times \nu(x), \quad x \in \partial \Omega,
$$

where $\mathbf{m}$ solves (3.13). 
Suppose (1.1) is satisfied, the magnetic field satisfies the following representation formula:

$$
\nabla \times \mathbf{H}(x) \times \nu(x)=\nabla \times \mathbf{H}_{0}(x) \times \nu(x)+\nabla \times \tilde{\mathbf{G}}_{D} \mathbf{j}(x) \times \nu(x), \quad x \in \partial \Omega,
$$

where $\mathbf{j}$ solves (3.13).

\section{$4 \quad$ Full asymptotic formula}

In this section we derive higher-order terms in our asymptotic expansions for the perturbed Maxwell's equations. As stated in the introduction, we restrict our derivation to the case of small inhomogeneity. We assume that this inhomogeneity $D$ has the form $D=\delta B+z$, where $z \in \Omega$ and $B$ is a bounded Lipschitz domain in $\mathbb{R}^{d}$ containing the origin.

Let us introduce the following Hilbert space:

$$
H_{0}(\operatorname{curl}, \Omega):=\left\{\mathbf{u} \in L^{2}(\Omega)^{3}: \quad \nabla \times \mathbf{u} \in L^{2}(\Omega)^{3}, \quad \nu \times u=0 \quad \text { on } \partial \Omega\right\} .
$$

It is well known that $H_{0}(\operatorname{curl}, \Omega)$ is a closed subspace of $H(\operatorname{curl}, \Omega)$ endowed with same norm.

The following result holds.

Theorem 4.1 Suppose (1.1) and (1.17) are satisfied. There exists $0<\delta_{0}$ such that, given an arbitrary $g \in T H_{\text {div }}^{-1 / 2}(\partial \Omega)$, and any $0<\delta<\delta_{0}$, the boundary value problem (1.9)-(1.14) has a unique (weak) solution $\mathbf{E}_{\delta}$. The constant $\delta_{0}$ depends on the domains $D, \Omega$, the medium parameters $\mu_{0}, \mu_{1}, \varepsilon_{0}, \varepsilon_{1}$, the frequency $\omega$, and $c_{0}$. Moreover, the following ascertions hold:

(i) The norms of the field $\mathbf{E}_{\delta} \in H(\mathrm{curl}, \Omega)$ are uniformly bounded:

$$
\exists C>0 ; \quad \forall \delta \in\left[0, \delta_{0}\right], \quad\left\|\mathbf{E}_{\delta}\right\|_{H(\operatorname{curl}, \Omega)} \leq C .
$$

(ii) The following convergence estimate holds:

$$
\exists C>0, \quad \forall \delta \in\left[0, \delta_{0}\right], \quad\left\|\mathbf{E}_{\delta}-\mathbf{E}_{0}\right\|_{H_{0}(\mathrm{curl}, \Omega)} \leq C \delta^{\frac{d}{2}} .
$$

The constant $C$ depends on the domains $B, \Omega$, the medium constants $\mu_{1}, \mu_{0}, \varepsilon_{1}, \varepsilon_{0}$ and $c_{0},\|\mathbf{g}\|_{T H_{d i v}^{-1 / 2}(\partial \Omega)}$, but is otherwise independent of parameter $\delta$.

Proof. The existence and uniqueness of solution to problem (1.9)-(1.14) can be followed from Theorem 1 of [4] when a similar study was done for magnetic field. Also, we refer the reader to same result found in [2] for time-harmonic Maxwell's equations with TE symmetry. 
(i) This uniform boundness can be deduced from the fact that $\mathbf{E}_{\delta} \rightarrow \mathbf{E}_{0}$ as $\delta \rightarrow 0$ and by replacing, in (3.18), $\mathbf{E}$ by $\mathbf{E}_{\delta}$ for $0 \leq \delta \leq \delta_{0}$.

(ii) Since both fields $\mathbf{E}_{\delta}$ and $\mathbf{E}_{0}$ satisfy the same boundary condition $\mathbf{E}_{\delta} \times \nu=$ $\mathbf{E}_{0} \times \nu=\mathbf{g}$ on $\partial \Omega$, the function $\mathbf{E}_{\delta}-\mathbf{E}_{0}$ belongs to $H_{0}(\operatorname{curl}, \Omega)$, and for any $\mathbf{v} \in H_{0}(\operatorname{curl}, \Omega):$

$$
\begin{aligned}
a_{\delta}\left(\mathbf{E}_{\delta}-\mathbf{E}_{0}, \mathbf{v}\right) & =\int_{\Omega} \frac{1}{\mu_{\delta}} \nabla \times\left(\mathbf{E}_{\delta}-\mathbf{E}_{0}\right) \cdot \nabla \times \mathbf{v}-\omega^{2} \int_{\Omega} \epsilon_{\delta}\left(\mathbf{E}_{\delta}-\mathbf{E}_{0}\right) \mathbf{v} \\
& =\int_{z+\delta B}\left[\left(\frac{1}{\mu_{0}}-\frac{1}{\mu_{1}}\right) \nabla \times \mathbf{E}_{0} \cdot \nabla \times \mathbf{v}+\omega^{2}\left(\epsilon_{1}-\epsilon_{0}\right) \mathbf{E}_{0} \mathbf{v}\right] d x
\end{aligned}
$$

where $0<\delta \leq \delta_{0}$. Subsequently, the term

$$
\left|\int_{z+\delta B}\left[\left(\frac{1}{\mu_{0}}-\frac{1}{\mu_{1}}\right) \nabla \times \mathbf{E}_{0} \cdot \nabla \times \mathbf{v}+\omega^{2}\left(\epsilon_{1}-\epsilon_{0}\right) \mathbf{E}_{0} \mathbf{v}\right] d x\right|
$$

is bounded by

$$
C\left(\left\|\mathbf{E}_{0}\right\|_{\mathbf{L}^{2}(z+\delta B)}+\left\|\nabla \times \mathbf{E}_{0}\right\|_{\mathbf{L}^{2}(z+\delta B)}\right)\|\mathbf{v}\|_{H(\operatorname{curl}, \Omega)} .
$$

In view of the fact that $D_{\delta}$ is bounded away from the boundary $\partial \Omega$ (see (1.1)), standard elliptic regularity results give that:

$$
\left\{\begin{array}{l}
\left\|\mathbf{E}_{0}\right\|_{\mathbf{L}^{\infty}\left(D_{\delta}\right)} \leq C\left\|\mathbf{E}_{0}\right\|_{H(\operatorname{curl}, \Omega)} \leq C\|\mathbf{g}\|_{T H_{\text {div }}^{-1 / 2}(\partial \Omega)}, \\
\left\|\nabla \times \mathbf{E}_{0}\right\|_{\mathbf{L}^{\infty}\left(D_{\delta}\right)} \leq C\left\|\mathbf{E}_{0}\right\|_{H(\operatorname{curl}, \Omega)} \leq C\|\mathbf{g}\|_{T H_{\text {div }}^{-1 / 2}(\partial \Omega)}
\end{array}\right.
$$

and so,

$$
\begin{gathered}
\left\|\mathbf{E}_{0}\right\|_{\mathbf{L}^{2}(z+\delta B)}+\left\|\nabla \times \mathbf{E}_{0}\right\|_{\mathbf{L}^{2}(z+\delta B)} \leq \delta^{\frac{d}{2}} \sqrt{|B|}\left(\left\|\mathbf{E}_{0}\right\|_{\mathbf{L}^{\infty}\left(D_{\delta}\right)}+\left\|\nabla \times \mathbf{E}_{0}\right\|_{\mathbf{L}^{\infty}\left(D_{\delta}\right)}\right) \\
\leq C \delta^{\frac{d}{2}} \sqrt{|B|}\|\mathbf{g}\|_{T H_{\text {div }}^{-1 / 2}(\partial \Omega)} .
\end{gathered}
$$

Now, replacing in the formulation (3.17) $\mathbf{E}$ by the difference $\mathbf{E}_{\delta}-\mathbf{E}$. It then follows from relation (3.18) that:

$$
\left\|\mathbf{E}_{\delta}-\mathbf{E}_{0}\right\|_{H_{0}(\operatorname{curl}, \Omega)} \leq C \delta^{\frac{d}{2}} \sqrt{|B|}\|\mathbf{g}\|_{T H_{\operatorname{div}}^{-1 / 2}(\partial \Omega)} .
$$

Consequently

$$
\left\|\mathbf{E}_{\delta}-\mathbf{E}_{0}\right\|_{H_{0}(\operatorname{curl}, \Omega)} \leq C \delta^{\frac{d}{2}}, \quad \text { for } 0 \leq \delta \leq \delta_{0},
$$

exactly as desired. 
To reveal the nature of the perturbations in the electric field, we introduce the local variables $y:=\frac{x-z}{\delta} \in B$ for $\delta \neq 0$ and $x \in \partial \Omega$. So that, for $y \in \partial D$ we may set:

$$
\left\{\begin{array}{l}
\mathbf{j}_{\delta}(y)=\mathbf{j}(z+\delta y) \\
\mathbf{m}_{\delta}(y)=\mathbf{m}(z+\delta y) \\
\mathcal{H}_{\delta}(y)=\mathcal{H}(z+\delta y)
\end{array}\right.
$$

Inserting the above notations into (3.13) we can show that, for $\delta$ small enough, the following estimate holds.

Proposition 4.2 Suppose that the domain $D=z+\delta B$ satisfies (1.1). Let $(\mathbf{j}, \mathbf{m}) \in$ $\mathbf{L}^{2}(\partial D) \times \mathbf{L}^{2}(\partial D)$ be the unique solution of (3.13). There exists $\delta_{1}>0$ such that for all $0<\delta \leq \delta_{1}$, there exists $C$ independent of $\delta$ such that:

$$
\|\mathbf{j}\|_{\mathbf{L}^{2}(\partial D)}+\|\mathbf{m}\|_{\mathbf{L}^{2}(\partial D)} \leq C\left(\delta^{-1}\|\mathcal{H} \times \nu\|_{\mathbf{L}^{2}(\partial D)}+\|\mathcal{H} \times \nu\|_{\mathbf{L}^{2}(\partial D)}\right) .
$$

Let $N \in \mathbb{N}$, and define

$$
H_{N}(x):=\sum_{|\zeta|=0}^{N} \frac{1}{\zeta !}\left(\partial^{\zeta} \mathcal{H}\right)(z)(x-z)^{\zeta}
$$

where $\mathcal{H}$ defined in (3.12). Regarding (3.13), we see that for $n \geq N$ there exists unique solution $\left(\mathbf{j}_{n}, \mathbf{m}_{n}\right)$ to the following system:

$$
\left\{\begin{array}{l}
\mathcal{V}_{D}^{k} \mathbf{j}_{n}(x)-\mathcal{V}_{D}^{k_{0}} \mathbf{m}_{n}(x)=\mathcal{H}_{n+1} \times \nu \text { on } \partial D \\
\frac{1}{\mu_{0}} \gamma_{N e u}\left(\mathcal{V}_{D}^{k} \mathbf{j}_{n}\right)-\frac{1}{\mu_{1}} \gamma_{N e u}\left(\mathcal{V}_{D}^{k_{0}} \mathbf{m}_{n}\right)=\frac{1}{\mu_{0}} \gamma_{\text {Neu }}\left(\mathcal{H}_{n+1}\right) \text { on } \partial D
\end{array}\right.
$$

Therefore, combining both relations (3.13) and (4.4) with (4.2), we immediately get for the obtained solution $\left(\mathbf{j}-\mathbf{j}_{n}, \mathbf{m}-\mathbf{m}_{n}\right) \in\left(\mathbf{L}^{2}(\partial D)\right)^{2}$ the following estimate:

$$
\begin{aligned}
\left\|\mathbf{j}-\mathbf{j}_{n}\right\|_{\mathbf{L}^{2}(\partial D)}+ & \left\|\mathbf{m}-\mathbf{m}_{n}\right\|_{\mathbf{L}^{2}(\partial D)} \leq C\left(\delta^{-1}\left\|\left(\mathcal{H}-\mathcal{H}_{n+1}\right) \times \nu\right\|_{\mathbf{L}^{2}(\partial D)}\right. \\
& \left.+\left\|\left(\operatorname{curl} \mathcal{H}-\operatorname{curl} \mathcal{H}_{n+1}\right) \times \nu\right\|_{\mathbf{L}^{2}(\partial D)}\right) .
\end{aligned}
$$

Let $\mathbf{E}_{0}$ be the solution of the background problem (1.15)-(1.16). Then, the pair $\left(\mathbf{j}_{0}=\frac{i}{\mu_{0} \omega} \nabla \times\left.\mathbf{E}_{0}\right|_{\partial \Omega} \times \nu, \mathbf{m}_{0}=-\left.\mathbf{E}_{0}\right|_{\partial \Omega} \times \nu\right)$ is well known and allows us to define the pair $(F, \tilde{F})$ of regular matrix valued function that satisfies:

$$
\begin{aligned}
\nabla \times \int_{\partial D} F(x, y) \mathbf{j}_{0}(y) d \sigma(y) \times \nu & =\mathbf{j}_{0}(x), \\
\int_{\partial D} \tilde{F}(x, y) \mathbf{m}_{0}(y) d \sigma(y) \times \nu & =\mathbf{m}_{0}(x) .
\end{aligned}
$$

Using above notations, the following uniform asymptotic expansion holds. 
Proposition 4.3 Let $\left(\mathbf{j}_{\delta}, \mathbf{m}_{\delta}\right)$ be defined by (4.1) where $(\mathbf{j}, \mathbf{m})$ solves (3.13). Then, $\left(\mathbf{j}_{\delta}, \mathbf{m}_{\delta}\right) \in T H_{\text {div }}^{-1 / 2}(\partial D) \times T H_{\text {div }}^{-1 / 2}(\partial D)$ is the unique solution to the following equations:

$$
\left\{\begin{array}{l}
\mathcal{V}_{B}^{k \delta} \mathbf{j}_{\delta}(y)-\mathcal{V}_{B}^{k_{0} \delta} \mathbf{m}_{\delta}(x)=\mathcal{H}_{\delta} \times \nu \quad \text { on } \partial B \\
\nabla \times\left(\frac{1}{\mu_{0}} \mathcal{V}_{B}^{k \delta} \mathbf{j}_{\delta}-\frac{1}{\mu_{1}} \mathcal{V}_{B}^{k_{0} \delta} \mathbf{m}_{\delta}\right) \times \nu=\frac{1}{\mu_{0}} \nabla \times \mathcal{H}_{\delta} \times \nu \quad \text { on } \partial B,
\end{array}\right.
$$

where the electromagnetic potentials $\mathcal{V}_{B}^{k \delta}$ and $\mathcal{V}_{B}^{k_{0} \delta}$ are defined by fundamental solutions $\Psi_{k \delta}$ and $\Psi_{k_{0} \delta}$, respectively.

Moreover, there exists $\delta_{2}>0$ such that the magnetic density $\mathbf{j}_{\delta}$ can be represented uniformly for $x \in \partial \Omega$ as follows:

$$
\begin{gathered}
\mathbf{j}_{\delta}(x)-\mathbf{j}_{0}(x)=\left(\frac{\mu_{0}}{\mu_{1}}-1\right) \nabla \times \int_{\partial D} F(x, y) \mathbf{j}_{0}(y) d \sigma(y) \times \nu \\
+\frac{i}{\mu_{1} \omega} \nabla \times \mathbf{G}_{D} \mathbf{m}_{n}(x) \times \nu(x)+O\left(\delta^{n+1}\right), \quad 0<\delta \leq \delta_{2},
\end{gathered}
$$

where $\mathbf{j}_{0}=\frac{i}{\mu_{0} \omega} \nabla \times\left.\mathbf{E}_{0}\right|_{\partial \Omega} \times \nu$ and $\mathbf{m}_{n}$ was defined in (4.4).

Suppose that above conditions are satisfied, the electric density $\mathbf{m}_{\delta}$ can be represented uniformly for $x \in \partial \Omega$ as follows:

$$
\begin{gathered}
\mathbf{m}_{\delta}(x)-\mathbf{m}_{0}(x)=\left(\frac{\varepsilon_{0}}{\varepsilon_{1}}-1\right) \int_{\partial D} \tilde{F}(x, y) \mathbf{m}_{0}(y) d \sigma(y) \times \nu \\
-\frac{i}{\varepsilon_{1} \omega} \nabla \times \tilde{\mathbf{G}}_{D} \mathbf{j}_{n}(x) \times \nu(x)+O\left(\delta^{n+1}\right), \quad 0<\delta \leq \delta_{2},
\end{gathered}
$$

where $\mathbf{m}_{0}=-\left.\mathbf{E}_{0}\right|_{\partial \Omega} \times \nu$ and $\mathbf{j}_{n}$ was defined in (4.4).

Proof. Let $\mathbf{m}_{n}$ be the solution of (4.4). Then, inserting the term $\nabla \times \mathbf{G}_{D} \mathbf{m}_{n}(x) \times$ $\nu(x)$ into relation (3.27), we immediately get for $x \in \partial \Omega$ :

$$
\begin{aligned}
\nabla \times \mathbf{E}_{\delta}(x) \times \nu(x)=\nabla & \times \mathbf{E}_{0}(x) \times \nu(x)+\nabla \times \mathbf{G}_{D}\left(\mathbf{m}-\mathbf{m}_{n}\right)(x) \times \nu(x) \\
& +\nabla \times \mathbf{G}_{D} \mathbf{m}_{n}(x) \times \nu(x) .
\end{aligned}
$$

In view of (1.5), we obtain that:

$$
\begin{gathered}
\mathbf{j}_{\delta}(x)-\frac{\mu_{0}}{\mu_{\delta}} \mathbf{j}_{0}(x)=\frac{i}{\mu_{\delta} \omega} \nabla \times \mathbf{G}_{D}\left(\mathbf{m}-\mathbf{m}_{n}\right)(x) \times \nu(x) \\
+\frac{i}{\mu_{\delta} \omega} \nabla \times \mathbf{G}_{D} \mathbf{m}_{n}(x) \times \nu(x) .
\end{gathered}
$$

As a consequence, 


$$
\begin{gathered}
\mathbf{j}_{\delta}(x)-\mathbf{j}_{0}(x)=\left(\frac{\mu_{0}}{\mu_{\delta}}-1\right) \mathbf{j}_{0}(x)+\frac{i}{\mu_{\delta} \omega} \nabla \times \mathbf{G}_{D}\left(\mathbf{m}-\mathbf{m}_{n}\right)(x) \times \nu(x) \\
+\frac{i}{\mu_{\delta} \omega} \nabla \times \mathbf{G}_{D} \mathbf{m}_{n}(x) \times \nu(x) .
\end{gathered}
$$

So that by integrating last relation in $D$, we get for $x \in \partial \Omega$ :

$$
\begin{gathered}
\mathbf{j}_{\delta}(x)-\mathbf{j}_{0}(x)=\left(\frac{\mu_{0}}{\mu_{1}}-1\right) \mathbf{j}_{0}(x)+\frac{i}{\mu_{1} \omega} \nabla \times \mathbf{G}_{D}\left(\mathbf{m}-\mathbf{m}_{n}\right)(x) \times \nu(x) \\
+\frac{i}{\mu_{1} \omega} \nabla \times \mathbf{G}_{D} \mathbf{m}_{n}(x) \times \nu(x) .
\end{gathered}
$$

Inserting (4.6) into relation (4.12), we immediately get:

$$
\begin{gathered}
\mathbf{j}_{\delta}(x)-\mathbf{j}_{0}(x)=\left(\frac{\mu_{0}}{\mu_{1}}-1\right) \nabla \times \int_{\partial D} F(x, y) \mathbf{j}_{0}(y) d \sigma(y) \times \nu \\
+\frac{i}{\mu_{1} \omega} \nabla \times \mathbf{G}_{D} \mathbf{m}_{n}(x) \times \nu(x)+\frac{i}{\mu_{1} \omega} \nabla \times \mathbf{G}_{D}\left(\mathbf{m}-\mathbf{m}_{n}\right)(x) \times \nu(x) .
\end{gathered}
$$

On the other hand, by relations (1.1) and (1.6) we naturally get:

$$
\left\|\frac{i}{\mu_{1} \omega} \nabla \times\left(\mathbf{G}_{D} \varphi\right) \times \nu\right\|_{\mathbf{L}^{\infty}(\partial \Omega \times \partial D)} \leq C\|\varphi\|_{\mathbf{L}^{2}(\partial D)},
$$

where $C$ is a positive constant independent of $\delta$. Replacing $\varphi$ by $\mathbf{m}-\mathbf{m}_{n}$, it then follows from (3.25) that:

$$
\left\|\frac{i}{\mu_{1} \omega} \nabla \times\left(\mathbf{G}_{D}\left(\mathbf{m}-\mathbf{m}_{n}\right)\right) \times \nu\right\|_{\mathbf{L}^{\infty}(\partial \Omega \times \partial D)} \leq C|\partial D|^{1 / 2}\left\|\mathbf{m}-\mathbf{m}_{n}\right\|_{\mathbf{L}^{2}(\partial D)} .
$$

To estimate the term $\left\|\mathbf{m}-\mathbf{m}_{n}\right\|_{\mathbf{L}^{2}(\partial D)}$, we may use firstly the definition of $\mathcal{H}_{n+1}$. So that,

$\left\|\mathcal{H} \times \nu-\mathcal{H}_{n+1} \times \nu\right\|_{\mathbf{L}^{2}(\partial D)} \leq C|\partial D|^{1 / 2}\left\|\mathcal{H} \times \nu-\mathcal{H}_{n+1} \times \nu\right\|_{\mathbf{L}^{\infty}(\partial D)} \leq C_{1}|\partial D|^{1 / 2} \delta^{n+2}\|\mathcal{H}\|_{C^{n+2}(\bar{D})}$.

Then, as before we obtain that:

$$
\begin{aligned}
\left\|\nabla \times\left(\mathcal{H}-\mathcal{H}_{n+1}\right) \times \nu\right\|_{L^{2}(\partial D)} & \leq \sqrt{|\partial D|}\left\|\nabla \times\left(\mathcal{H}-\mathcal{H}_{n+1}\right) \times \nu\right\|_{L^{\infty}(\partial D)} \\
& \leq\|\mathcal{H}\|_{\mathcal{C}^{n+1}(\bar{D})}|x-z|^{n+1} \sqrt{|\partial D|} \\
& \leq C_{2} \delta^{n+1} \sqrt{|\partial D|}\|\mathcal{H}\|_{\mathcal{C}^{n+1}(\bar{D})} .
\end{aligned}
$$

Thus, by Theorem (3.5) and relation (4.5) we immediately get:

$$
\left\|\mathbf{m}-\mathbf{m}_{n}\right\|_{L^{2}(\partial D)} \leq\left(C_{1} c_{n+2}+C_{2} c_{n+1}\right)\|\mathbf{g}\|_{T H_{\mathrm{div}}^{-1 / 2}(\partial \Omega)} \delta^{n+1} \sqrt{|\partial D|} .
$$


So that,

$$
\left\|\mathbf{m}-\mathbf{m}_{n}\right\|_{L^{2}(\partial D)} \leq c_{n}^{\prime} \sqrt{|\partial D|} \delta^{n+1} .
$$

Finally, inserting (4.15) into (4.14) we get for all $x \in \partial \Omega$ that:

$$
\left\|\frac{i}{\mu_{1} \omega} \nabla \times \mathbf{G}_{D}\left(\mathbf{m}-\mathbf{m}_{n}\right)(x) \times \nu(x)\right\|_{L^{\infty}(\partial \Omega \times \partial D)} \leq c_{n}^{\prime} \delta^{n+1}|\partial D|=C_{n} \delta^{n+1} .
$$

The proof of (4.9) is achieved by inserting last estimation into relation (4.13). Formula (4.10) for the density $\mathbf{m}_{\delta}$ follows in a manner completely similar to that for (4.9).

The main result of this paper is the following full asymptotic expansion of the tangential trace of electromagnetic fields.

Theorem 4.4 Suppose that the domain $D=z+\delta B$ satisfies (1.1), and assume that we have all hypothesis of Proposition 4.3. Let $\mathbf{E}_{\delta}$ be the solution of (1.9)-(1.14) and suppose that condition (1.17) is well satisfied. The following pointwise asymptotic expansion, for the electric field, on $\partial \Omega$ holds for $d=2,3$ :

$$
\begin{gathered}
\left(\mathbf{E}_{\delta}(x)-\mathbf{E}_{0}(x)\right) \times \nu(x)= \\
\left(1-\frac{\varepsilon_{0}}{\varepsilon_{1}}\right) \sum_{|p|=0}^{n} \frac{1}{p !} \delta^{|p|} \partial_{z}^{p} \tilde{F}(x, z) \int_{\partial B} t^{p} \mathbf{m}_{0}(t) d \sigma(t) \times \nu(x) \\
+\frac{1}{\epsilon_{1} \omega} \delta^{d-1} \sum_{|p|=0}^{n+1} \sum_{|q|=0}^{n+1-|p|} \frac{1}{p ! q !} \delta^{|p|+|q|}\left(\partial^{p} \mathcal{H}\right)(z) \partial_{z}^{q} \nabla \times\left(\tilde{\mathbf{G}}_{D} \mathbf{a}_{p q}\right) \times \nu(x)+O\left(\delta^{n+1}\right),
\end{gathered}
$$

with $p, q \in \mathbb{N}^{d}$ are multi-index and $\mathbf{a}_{p q}=i \int_{\partial B} \mathbf{j}_{q}(t) t^{p} d \sigma(t)$.

Suppose above conditions are satisfied, the magnetic field satisfies the following asymptotic expansion formula, for $x \in \partial \Omega$ :

$$
\begin{gathered}
\left(\mathbf{H}_{\delta}(x)-\mathbf{H}_{0}(x)\right) \times \nu(x)= \\
\left(1-\frac{\mu_{0}}{\mu_{1}}\right) \sum_{|p|=0}^{n} \frac{1}{p !} \delta^{|p|} \nabla \times \partial_{z}^{p} F(x, z) \int_{\partial B} t^{p} \mathbf{j}_{0}(t) d \sigma(t) \times \nu(x) \\
-\frac{1}{\mu_{1} \omega} \delta^{d-1} \sum_{|p|=0}^{n+1} \sum_{|q|=0}^{n-|p|+1} \frac{1}{p ! q !} \delta^{|p|+|q|}\left(\partial^{p} \mathcal{H}\right)(z) \partial_{z}^{q} \nabla \times\left(\mathbf{G}_{D} \mathbf{b}_{p q}\right) \times \nu(x)+O\left(\delta^{n+1}\right),
\end{gathered}
$$

where $\mathbf{b}_{p q}=i \int_{\partial B} \mathbf{m}_{q}(t) t^{p} d \sigma(t)$. In both asymptotic formulae, the term $O\left(\delta^{n+1}\right)$ is dominated by $C \delta^{n+1}\|\mathbf{g}\|_{T H_{d i v}^{-1 / 2}(\partial \Omega)}$ for some $C$ independent of $x \in \partial \Omega$. 
Proof. The proofs of (4.16) and (4.17) can be done by a similar way. So that, we may only prove (4.17). Let $\zeta \in \mathbb{N}^{d}$ be a multi-index. Since $D=\delta B+z$, one can use the change of variables $y:=\frac{x-z}{\delta}(\delta \neq 0)$ to expand $\mathbf{G}^{\mathbf{0}}(x, \delta y+z)$ asymptotically as $\delta \rightarrow 0$.

Then, for $x$ near $\partial \Omega, z \in \Omega$ and $t \in \partial B$ and for $\delta$ sufficiently small, we get:

$$
\mathbf{G}^{\mathbf{0}}(x, \delta t+z):=\sum_{|\zeta|=0}^{\infty} \frac{\delta^{|\zeta|}}{\zeta !} \partial_{z}^{\zeta} \mathbf{G}^{\mathbf{0}}(x, z) t^{\zeta}
$$

So that, from (3.25) we obtain:

$$
\mathbf{G}_{D}\left(\varphi\left(\frac{x-z}{\delta}\right)\right):=\sum_{|\zeta|=0}^{\infty} \frac{\delta^{|\zeta|+d-1}}{\zeta !} \partial_{z}^{\zeta} \mathbf{G}^{\mathbf{0}}(x, z) \int_{\partial B} \varphi(t) t^{\zeta} d \sigma(t) .
$$

Now let $\left(\mathbf{j}_{\zeta}, \mathbf{m}_{\zeta}\right)$ be the solution of:

$$
\left\{\begin{array}{l}
\mathcal{V}_{B}^{k \delta} \mathbf{j}_{\zeta}(x)-\mathcal{V}_{B}^{k_{0} \delta} \mathbf{m}_{\zeta}(x)=\nu \times Z_{\zeta} \quad \text { on } \partial B \\
\frac{1}{\mu_{0}} \gamma_{N e u}\left(\mathcal{V}_{B}^{k \delta} \mathbf{j}_{\zeta}\right)-\frac{1}{\mu_{1}} \gamma_{N e u}\left(\mathcal{V}_{B}^{k_{0} \delta} \mathbf{m}_{\zeta}\right)=\frac{1}{\mu_{0}} \gamma_{N e u}\left(Z_{\zeta}\right) \text { on } \partial B
\end{array}\right.
$$

where $Z_{\zeta}=x^{\zeta}$, and $\zeta$ is a multi-index. If $\left(\mathbf{j}_{n}, \mathbf{m}_{n}\right)$ is the solution of problem (4.4), then one can use definition (4.3) to expand:

$$
\mathbf{m}_{n}(x)=\sum_{|\zeta|=0}^{n+1} \frac{\delta^{|\zeta|}}{\zeta !} \partial^{\zeta} \mathcal{H}(z) \mathbf{m}_{\zeta}\left(\frac{x-z}{\delta}\right)
$$

The density $\mathbf{j}_{n}$ can be expressed analogously. On other hand, for $x$ near $\partial \Omega, z \in \Omega$ and $t \in \partial B$ and for $\delta$ sufficiently small, we have:

$$
F(x, \delta t+z):=\sum_{|\zeta|=0}^{\infty} \frac{\delta^{|\zeta|}}{\zeta !} \partial_{z}^{\zeta} F(x, z) t^{\zeta}
$$

Therefore,

$$
\begin{gathered}
\left.\int_{\partial D} F(x, y) \mathbf{j}_{0}(y) d \sigma(y)=\int_{\partial D} F(x, \delta t+z) \mathbf{j}_{0}\left(\frac{x-z}{\delta}\right)\right) d \sigma(t) \\
=\sum_{|\zeta|=0}^{\infty} \frac{\delta^{|\zeta|}}{\zeta !} \partial_{z}^{\zeta} F(x, z) \int_{\partial B} \mathbf{j}_{0}(t) t^{\zeta} d \sigma(t) .
\end{gathered}
$$


Now inserting both relations (4.21) and (4.22) into the expansion (4.9), we immediately get for $x \in \partial \Omega$ :

$$
\begin{gathered}
\mathbf{j}_{\delta}(x)=\mathbf{j}_{0}(x)+\left(\frac{\mu_{0}}{\mu_{1}}-1\right) \sum_{|p|=0}^{n} \frac{1}{p !} \delta^{|p|} \nabla \times \partial_{z}^{p} F(x, z) \int_{\partial B} t^{p} \mathbf{j}_{0}(t) d \sigma(t) \times \nu(x) \\
+\frac{i}{\mu_{1} \omega} \sum_{|\zeta|=0}^{n+1} \frac{\delta^{|\zeta|}}{\zeta !} \partial^{\zeta} \mathcal{H}(z) \nabla \times \mathbf{G}_{D}\left(\mathbf{m}_{\zeta}\left(\frac{x-z}{\delta}\right)\right) \times \nu(x)+O\left(\delta^{n+1}\right)
\end{gathered}
$$

Observe that $\mathbf{j}_{\delta}=\nu \times \mathbf{H}_{\delta}$ and $\mathbf{j}_{0}=\nu \times \mathbf{H}_{0}$, then we obtain:

$$
\begin{gathered}
\mathbf{H}_{\delta}(x) \times \nu(x)=\mathbf{H}_{0}(x) \times \nu(x)+\left(1-\frac{\mu_{0}}{\mu_{1}}\right) \sum_{|p|=0}^{n} \frac{1}{p !} \delta^{|p|} \nabla \times \partial_{z}^{p} F(x, z) \int_{\partial B} t^{p} \mathbf{j}_{0}(t) d \sigma(t) \times \nu(x) \\
-\frac{i}{\omega \mu_{1}} \sum_{|\zeta|=0}^{n+1} \frac{\delta^{|\zeta|}}{\zeta !} \partial^{\zeta} \mathcal{H}(z) \nabla \times \mathbf{G}_{D}\left(\mathbf{m}_{\zeta}\left(\frac{x-z}{\delta}\right)\right) \times \nu(x)+O\left(\delta^{n+1}\right) .
\end{gathered}
$$

To achieve the proof, we insert (4.19) into uniform expansion (4.24) and we put $\mathbf{b}_{p q}=i \int_{\partial B} \mathbf{m}_{q}(t) t^{p} d \sigma(t)$.

As a consequence of previous results, we have the following complete asymptotic expansions.

Corollary 4.5 Suppose that we have all hypothesis of Theorem 4.4. The following pointwise asymptotic expansion on $\partial \Omega$ holds for $d=2,3$ :

$$
\begin{gathered}
\nabla \times \mathbf{E}_{\delta}(x) \times \nu(x)=\frac{\mu_{1}}{\mu_{0}} \nabla \times \mathbf{E}_{0}(x) \times \nu(x)+ \\
i \omega\left(\mu_{1}-\mu_{0}\right) \sum_{|p|=0}^{n} \frac{1}{p !} \delta^{|p|} \nabla \times \partial_{z}^{p} F(x, z) \int_{\partial B} t^{p} \mathbf{j}_{0}(t) d \sigma(t) \times \nu(x) \\
-i \delta^{d-1} \sum_{|p|=0}^{n+1} \sum_{|q|=0}^{n-|p|+1} \frac{1}{p ! q !} \delta^{|p|+|q|}\left(\partial^{p} \mathcal{H}\right)(z) \partial_{z}^{q} \nabla \times\left(\mathbf{G}_{D} \mathbf{b}_{p q}\right) \times \nu(x)+O\left(\delta^{n+1}\right),
\end{gathered}
$$

where $\mathbf{b}_{p q}=i \int_{\partial B} \mathbf{m}_{q}(t) t^{p} d \sigma(t)$. In both asymptotic formulae, the term $O\left(\delta^{n+1}\right)$ is dominated by $C \delta^{n+1}\|\mathbf{g}\|_{T H_{d i v}^{-1 / 2}(\partial \Omega)}$ for some $C$ independent of $x \in \partial \Omega$. 

$\mathcal{V}_{D}^{k}$.

We now proceed to construct an asymptotic expansion of the electric potential Recall from (2.5) that,

$$
\mathcal{V}_{D}^{k} \varphi=k \mathcal{S}_{D}^{k} \varphi+1 / k \nabla \mathcal{S}_{D}^{k} \operatorname{div} \partial \Omega \varphi
$$

where $\varphi \in \mathbf{L}^{2}(\partial D)$ is a given density. According to (2.3) and Remark 2.1, we have:

$$
\mathcal{S}_{D}^{k} \varphi(x)=\int_{\partial D} \Phi_{k}(x, y) \varphi(y) d \sigma(y) .
$$

Therefore,

$$
\mathcal{S}_{D}^{k} \varphi(x)=\int_{\partial D} \Phi_{k}(x, y) \varphi\left(\frac{y-z}{\delta}\right) d \sigma(y)=\delta^{d-1} \int_{\partial B} \Phi_{k}(x, z+\delta t) \varphi(t) d \sigma(t) .
$$

On other hand, for $x$ near $\partial \Omega, z \in \Omega$ and $t \in \partial B$ and for $\delta$ sufficiently small, we have:

$$
\Phi_{k}(x, \delta t+z):=\sum_{|\zeta|=0}^{\infty} \frac{\delta^{|\zeta|}}{\zeta !} \partial_{z}^{\zeta} \Phi_{k}(x, z) t^{\zeta}
$$

Then,

$$
\mathcal{S}_{D}^{k} \varphi(x)=\sum_{|p|=0}^{\infty} \frac{\delta^{|p|+d-1}}{p !} \partial_{z}^{p} \Phi_{k}(x, z) \int_{\partial B} t^{p} \varphi(t) d \sigma(t) .
$$

Consequently,

$$
\begin{gathered}
\mathcal{V}_{D}^{k} \varphi(x)=\sum_{|p|=0}^{\infty} \frac{\delta^{|p|+d-1}}{p !}\left[k \partial_{z}^{p} \Phi_{k}(x, z) \int_{\partial B} t^{p} \varphi(t) d \sigma(t)\right. \\
\left.+1 / k \nabla \partial_{z}^{p} \Phi_{k}(x, z) \int_{\partial B} t^{p} \operatorname{div}{ }_{\partial B} \varphi(t) d \sigma(t)\right] .
\end{gathered}
$$

Now, we state the following complete asymptotic expansion in $\Omega$.

Theorem 4.6 Suppose that we have all hypothesis of Theorem 4.4. Let $\mathbf{G}^{0}$ be the solution of (3.24). Then, the following uniform asymptotic expansion for $x \in \Omega$ holds:

$$
\begin{aligned}
\mathbf{E}_{\delta}(x) & =\mathbf{E}_{0}(x)-\delta^{d}\left[\left(1-\frac{\mu_{1}}{\mu_{0}}\right) \mathbf{h}_{1}(\delta ; x, z)+\omega^{2}\left({ }_{1}-{ }_{0}\right) \mathbf{e}_{1}(\delta ; x, z)\right] \\
& +\delta^{2 d-1}\left[\left(1-\frac{\mu_{1}}{\mu_{0}}\right) \mathbf{h}_{2}(\delta ; x, z)+\omega^{2}\left({ }_{1}-0\right) \mathbf{e}_{2}(\delta ; x, z)\right] \\
& -\delta^{3 d-2}\left[\left(1-\frac{\mu_{1}}{\mu_{0}}\right) \mathbf{h}_{3}(\delta ; x, z)+\omega^{2}\left({ }_{1}-0\right) \mathbf{e}_{3}(\delta ; x, z)\right]+O\left(\delta^{n+1}\right),
\end{aligned}
$$

where for $i \in\{1,2,3\}$, the functions $\left(\mathbf{e}_{i}, \mathbf{h}_{i}\right) \in[H(\text { curl }, \Omega)]^{2}$ are analytic with respect to $\delta$ and satisfy $\mathbf{e}_{i}(0 ; x, z) \neq 0, \mathbf{h}_{i}(0 ; x, z) \neq 0$. The term $O\left(\delta^{n+1}\right)$ is dominated by $C \delta^{n+1}\|\mathbf{g}\|_{T H_{\text {div }}^{-1 / 2}(\partial \Omega)}$ for some positive constant $C$ independent of the geometry of the medium . 
Proof. First of all, we note that the problem (3.24) has a unique solution since $\omega$ is assumed not to be a resonant frequency for the problem (1.15)-(1.16). For any $x \in \Omega$ we now get, from use of (3.24) and integration by parts,

$$
\begin{aligned}
\mathbf{E}_{\delta}(x) & =\mathbf{E}_{0}(x)+i \omega\left(1-\frac{\mu_{1}}{\mu_{0}}\right) \int_{z+\delta B} \nabla_{y} \times \mathbf{G}^{\mathbf{0}}(x, y) \mathbf{H}_{\delta}(y) d y \\
& -\omega^{2}\left(\varepsilon_{1}-\varepsilon_{0}\right) \int_{z+\delta B} \mathbf{G}^{\mathbf{0}}(x, y) \mathbf{E}_{\delta}(y) d y
\end{aligned}
$$

Based on Theorem 3.5, we may use (4.27) to develop the desired asymptotic formula for all $x \in \Omega$.

Before formulating this, we need to develop the following:

$$
\int_{z+\delta B} \nabla_{y} \times \mathbf{G}^{\mathbf{0}}(x, y) \psi(y) d y=\delta^{d} \int_{B} \nabla_{t} \times \mathbf{G}^{0}(x, z+\delta t) \psi(t) d t
$$

Using expansion (4.18), we obtain:

$$
\int_{z+\delta B} \nabla_{y} \times \mathbf{G}^{\mathbf{0}}(x, y) \psi(y) d y:=\sum_{|\zeta|=0}^{\infty} \frac{\delta^{|\zeta|+d}}{\zeta !} \nabla_{z} \times \partial_{z}^{\zeta} \mathbf{G}^{\mathbf{0}}(x, z) \int_{B} \psi(t) t^{\zeta} d t
$$

Similarly,

$$
\int_{z+\delta B} \mathbf{G}^{\mathbf{0}}(x, y) \psi(y) d y:=\sum_{|\zeta|=0}^{\infty} \frac{\delta^{|\zeta|+d}}{\zeta !} \partial_{z}^{\zeta} \mathbf{G}^{\mathbf{0}}(x, z) \int_{B} \psi(t) t^{\zeta} d t .
$$

Now using representation (3.14), we get for $x \in D$ that

$$
\mathbf{E}_{\delta}(x)=\mathcal{V}_{D}^{k}\left(\mathbf{j}_{\delta}\right)(x) .
$$

So that by (4.26), we get for $x \in D$ :

$\mathbf{E}_{\delta}(x)=\sum_{|p|=0}^{\infty} \frac{\delta^{|p|}}{p !}\left[k \partial_{z}^{p} \Phi_{k}(x, z) \int_{\partial B} t^{p} \mathbf{j}_{\delta}(t) d \sigma(t)+1 / k \nabla \partial_{z}^{p} \Phi_{k}(x, z) \int_{\partial B} t^{p} \operatorname{div} \partial B\left(\mathbf{j}_{\delta}\right)(t) d \sigma(t)\right]$.

Consequently, by using relation (4.23) we obtain:

$$
\begin{aligned}
& \mathbf{E}_{\delta}(x)=\sum_{|p|=0}^{n+1} \frac{\delta^{|p|}}{p !}\left[k \partial_{z}^{p} \Phi_{k}(x, z) \int_{\partial B} t^{p} \mathbf{j}_{0}(t) d \sigma(t)+1 / k \nabla \partial_{z}^{p} \Phi_{k}(x, z) \int_{\partial B} t^{p} \operatorname{div}{ }_{\partial B}\left(\mathbf{j}_{0}\right)(t) d \sigma(t)\right] \\
& -\left(1-\frac{\mu_{0}}{\mu_{1}}\right) \delta^{d-1} \sum_{|p|=0}^{n+1} \sum_{|q|=0}^{n+1-|p|} \frac{\delta^{|p|+|q|}}{p ! q !}\left[k \partial_{z}^{p} \Phi_{k}(x, z) \int_{\partial B} t^{p}\left(\nabla \times \partial_{z}^{q} F(t, z) \int_{\partial B} s^{q} \mathbf{j}_{0}(s) d \sigma(s) \times \nu(t)\right) d \sigma(t)\right.
\end{aligned}
$$




$$
\begin{gathered}
\left.+1 / k \nabla \partial_{z}^{p} \Phi_{k}(x, z) \int_{\partial B} t^{p} \operatorname{div} \partial B\left(\nabla \times \partial_{z}^{q} F(t, z) \int_{\partial B} s^{q} \mathbf{j}_{0}(s) d \sigma(s) \times \nu(t)\right) d \sigma(t)\right] \\
+\frac{1}{\mu_{1} \omega} \delta^{2 d-2} \sum_{|p|=0}^{n+1} \sum_{|q|=0}^{n+1-|p|} \sum_{|\zeta|=0}^{n+1-|p|-|q|} \frac{\delta^{|p|+|q|+|\zeta|}}{p ! q ! \zeta !}\left[k \partial_{z}^{p} \Phi_{k}(x, z) \int_{\partial B} t^{p}\left(\partial^{q} \mathcal{H}\right)(z) \partial_{z}^{\zeta} \nabla \times\left(\mathbf{G}_{D} \mathbf{b}_{q \zeta}\right) \times \nu(t)\right) d \sigma(t) \\
\left.\left.+1 / k \nabla \partial_{z}^{p} \Phi_{k}(x, z) \int_{\partial B} t^{p} \operatorname{div}{ }_{\partial B}\left(\partial^{q} \mathcal{H}\right)(z) \partial_{z}^{\zeta} \nabla \times\left(\mathbf{G}_{D} \mathbf{b}_{q \zeta}\right) \times \nu(t)\right) d \sigma(t)\right]+O\left(\delta^{n+1}\right) .
\end{gathered}
$$

Therefore, inserting (4.30) into (4.29), we get:

$$
\begin{aligned}
& \int_{z+\delta B} \mathbf{G}^{\mathbf{0}}(x, y) \mathbf{E}_{\delta}(y) d y=\delta^{d} \sum_{|\alpha|=0}^{n+1} \sum_{|p|=0}^{n+1-|\alpha|} \frac{\delta^{|\alpha|+|p|}}{\alpha ! p !} \partial_{z}^{\alpha} \mathbf{G}^{\mathbf{0}}(x, z) \int_{B}\left\{k \partial_{z}^{p} \Phi_{k}(t, z) \int_{\partial B} s^{p} \mathbf{j}_{0}(s) d \sigma(s)\right. \\
& \left.+1 / k \nabla \partial_{z}^{p} \Phi_{k}(t, z) \int_{\partial B} s^{p} \operatorname{div}{ }_{\partial B}\left(\mathbf{j}_{0}\right)(s) d \sigma(s)\right\}(t) t^{\alpha} d t \\
& -\left(1-\frac{\mu_{0}}{\mu_{1}}\right) \delta^{2 d-1} \sum_{|\alpha|=0}^{n+1} \sum_{|p|=0}^{n+1-|\alpha|} \sum_{|q|=0}^{n+1-|\alpha|-|p|} \frac{\delta^{|\alpha|+|p|+|q|}}{\alpha ! p ! q !} \partial_{z}^{\alpha} \mathbf{G}^{\mathbf{0}}(x, z) \int_{B}\left\{k \partial_{z}^{p} \Phi_{k}(t, z) \times\right. \\
& \int_{\partial B} s^{p}\left(\nabla \times \partial_{z}^{q} F(s, z) \int_{\partial B} \tau^{q} \mathbf{j}_{0}(\tau) d \sigma(\tau) \times \nu(s)\right) d \sigma(s) \\
& \left.+1 / k \nabla \partial_{z}^{p} \Phi_{k}(t, z) \int_{\partial B} s^{p} \operatorname{div}{ }_{\partial B}\left(\nabla \times \partial_{z}^{q} F(s, z) \int_{\partial B} \tau^{q} \mathbf{j}_{0}(\tau) d \sigma(\tau) \times \nu(s)\right) d \sigma(s)\right\}(t) t^{\alpha} d t
\end{aligned}
$$

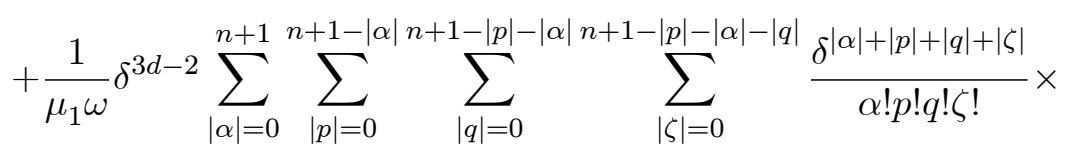

$$
\begin{aligned}
& \partial_{z}^{\alpha} \mathbf{G}^{\mathbf{0}}(x, z) \int_{B}\left\{k \partial_{z}^{p} \Phi_{k}(t, z) \int_{\partial B} s^{p}\left(\partial^{q} \mathcal{H}\right)(z) \partial_{z}^{\zeta} \nabla \times\left(\mathbf{G}_{D} \mathbf{b}_{q \zeta}\right) \times \nu(s)\right) d \sigma(s) \\
& \left.\left.+1 / k \nabla \partial_{z}^{p} \Phi_{k}(t, z) \int_{\partial B} s^{p} \operatorname{div}{ }_{\partial B}\left(\partial^{q} \mathcal{H}\right)(z) \partial_{z}^{\zeta} \nabla \times\left(\mathbf{G}_{D} \mathbf{b}_{q \zeta}\right) \times \nu(s)\right) d \sigma(s)\right\}(t) t^{\alpha} d t \\
& +O\left(\delta^{n+1}\right) \text {. }
\end{aligned}
$$

To simplify the above formulations, we may define:

$$
\begin{gathered}
\mathbf{e}_{\alpha p}(x, z):=\partial_{z}^{\alpha} \mathbf{G}^{\mathbf{0}}(x, z) \int_{B}\left\{k \partial_{z}^{p} \Phi_{k}(t, z) \int_{\partial B} s^{p} \mathbf{j}_{0}(s) d \sigma(s)\right. \\
\left.+1 / k \nabla \partial_{z}^{p} \Phi_{k}(t, z) \int_{\partial B} s^{p} \operatorname{div} \partial B\left(\mathbf{j}_{0}\right)(s) d \sigma(s)\right\}(t) t^{\alpha} d t
\end{gathered}
$$




$$
\begin{gathered}
\mathbf{e}_{\alpha p q}(x, z):=\left(1-\frac{\mu_{0}}{\mu_{1}}\right) \partial_{z}^{\alpha} \mathbf{G}^{\mathbf{0}}(x, z) \int_{B}\left\{k \partial_{z}^{p} \Phi_{k}(t, z) \times\right. \\
\int_{\partial B} s^{p}\left(\nabla \times \partial_{z}^{q} F(s, z) \int_{\partial B} \tau^{q} \mathbf{j}_{0}(\tau) d \sigma(\tau) \times \nu(s)\right) d \sigma(s) \\
\left.+1 / k \nabla \partial_{z}^{p} \Phi_{k}(t, z) \int_{\partial B} s^{p} \operatorname{div}{ }_{\partial B}\left(\nabla \times \partial_{z}^{q} F(s, z) \int_{\partial B} \tau^{q} \mathbf{j}_{0}(\tau) d \sigma(\tau) \times \nu(s)\right) d \sigma(s)\right\}(t) t^{\alpha} d t
\end{gathered}
$$

and

$$
\begin{aligned}
& \mathbf{e}_{\alpha p q \zeta}(x, z):=\frac{1}{\mu_{1} \omega} \partial_{z}^{\alpha} \mathbf{G}^{\mathbf{0}}(x, z) \int_{B}\left\{k \partial_{z}^{p} \Phi_{k}(t, z) \int_{\partial B} s^{p}\left(\partial^{q} \mathcal{H}\right)(z) \partial_{z}^{\zeta} \nabla \times\left(\mathbf{G}_{D} \mathbf{b}_{q \zeta}\right) \times \nu(s)\right) d \sigma(s) \\
& \left.\left.\quad+1 / k \nabla \partial_{z}^{p} \Phi_{k}(t, z) \int_{\partial B} s^{p} \operatorname{div}{ }_{\partial B}\left(\partial^{q} \mathcal{H}\right)(z) \partial_{z}^{\zeta} \nabla \times\left(\mathbf{G}_{D} \mathbf{b}_{q \zeta}\right) \times \nu(s)\right) d \sigma(s)\right\}(t) t^{\alpha} d t .
\end{aligned}
$$

Consequently, (4.31) becomes:

$$
\begin{gathered}
\int_{z+\delta B} \mathbf{G}^{\mathbf{0}}(x, y) \mathbf{E}_{\delta}(y) d y=\delta^{d} \sum_{|\alpha|=0}^{n+1} \sum_{|p|=0}^{n+1-|\alpha|} \frac{\delta^{|\alpha|+|p|}}{\alpha ! p !} \mathbf{e}_{\alpha p}(x, z) \\
-\delta^{2 d-1} \sum_{|\alpha|=0}^{n+1} \sum_{|p|=0}^{n+1-|\alpha| n+1-|\alpha|-|p|} \sum_{|q|=0}^{n} \frac{\delta^{|\alpha|+|p|+|q|}}{\alpha ! p ! q !} \mathbf{e}_{\alpha p q}(x, z) \\
+\delta^{3 d-2} \sum_{|\alpha|=0}^{n+1} \sum_{|p|=0}^{n+1-|\alpha| n+1-|p|-|\alpha| n+1-|p|-|\alpha|-|q|} \sum_{|q|=0}^{n} \frac{\delta^{|\alpha|+|p|+|q|+|\zeta|}}{\alpha ! p ! q ! \zeta !} \mathbf{e}_{\alpha p q \zeta}(x, z)+O\left(\delta^{n+1}\right) .
\end{gathered}
$$

Using representation (3.23), we obtain:

$$
\mathbf{H}_{\delta}(x)=\frac{1}{i \mu_{1} \omega} \nabla \times \mathcal{V}_{D}^{k}\left(\mathbf{j}_{\delta}\right)(x), \quad x \in D .
$$

It then follows from (4.30) and (4.28) that:

$$
\begin{gathered}
\int_{z+\delta B} \nabla_{y} \times \mathbf{G}^{\mathbf{0}}(x, y) \mathbf{H}_{\delta}(y) d y=-\delta^{d} \sum_{|\alpha|=0}^{n+1} \sum_{|p|=0}^{n+1-|\alpha|} \frac{\delta^{|\alpha|+|p|}}{\alpha ! p !} \mathbf{h}_{\alpha p}(x, z) \\
+\delta^{2 d-1} \sum_{|\alpha|=0}^{n+1} \sum_{|p|=0}^{n+1-|\alpha|} \sum_{|q|=0}^{n+1-|\alpha|-|p|} \frac{\delta^{|\alpha|+|p|+|q|}}{\alpha ! p ! q !} \mathbf{h}_{\alpha p q}(x, z) \\
-\delta^{3 d-2} \sum_{|\alpha|=0}^{n+1} \sum_{|p|=0}^{n+1-|\alpha|} \sum_{|q|=0}^{n+1-|p|-|\alpha|} \times
\end{gathered}
$$




$$
\sum_{|\zeta|=0}^{n+1-|p|-|\alpha|-|q|} \frac{\delta^{|\alpha|+|p|+|q|+|\zeta|}}{\alpha ! p ! q ! \zeta !} \mathbf{h}_{\alpha p q \zeta}(x, z)+O\left(\delta^{n+1}\right) .
$$

When we have defined:

$$
\left\{\begin{array}{l}
\mathbf{h}_{\alpha p}(x, z):=\frac{i}{\mu_{1} \omega} \nabla_{x} \times\left(\nabla_{z} \times \mathbf{e}_{\alpha p}(x, z)\right), \\
\mathbf{h}_{\alpha p q}(x, z):=\frac{i}{\mu_{1} \omega} \nabla_{x} \times\left(\nabla_{z} \times \mathbf{e}_{\alpha p q}(x, z)\right), \\
\mathbf{h}_{\alpha p q \zeta}(x, z):=\frac{i}{\mu_{1} \omega} \nabla_{x} \times\left(\nabla_{z} \times \mathbf{e}_{\alpha p q \zeta}(x, z)\right) .
\end{array}\right.
$$

Inserting both relations (4.32) and (4.33) into (4.27). We get:

$$
\begin{aligned}
\mathbf{E}_{\delta}(x) & =\mathbf{E}_{0}(x)-\delta^{d} \sum_{|\alpha|=0}^{n+1} \sum_{|p|=0}^{n+1-|\alpha|} \frac{\delta^{|\alpha|+|p|}}{\alpha ! p !}\left[\omega\left(1-\frac{\mu_{1}}{\mu_{0}}\right) \mathbf{h}_{\alpha p}(x, z)+\omega^{2}\left(\varepsilon_{1}-\varepsilon_{0}\right) \mathbf{e}_{\alpha p}(x, z)\right] \\
& +\delta^{2 d-1} \sum_{|\alpha|=0}^{n+1} \sum_{|p|=0}^{n+1-|\alpha|} \sum_{|q|=0}^{n+1-|\alpha|-|p|} \frac{\delta^{|\alpha|+|p|+|q|}}{\alpha ! p ! q !}\left[\omega\left(1-\frac{\mu_{1}}{\mu_{0}}\right) \mathbf{h}_{\alpha p q}(x, z)+\omega^{2}\left(\varepsilon_{1}-\varepsilon_{0}\right) \mathbf{e}_{\alpha p q}(x, z)\right] \\
& -\delta^{3 d-2} \sum_{|\alpha|=0}^{n+1} \sum_{|p|=0}^{n+1-|\alpha|} \sum_{|q|=0}^{n+1-|\alpha|-|p|} \sum_{|\zeta|=0}^{n+1-|\alpha|-|p|-|q|} \frac{\delta^{|\alpha|+|p|+|q|+|\zeta|}}{\alpha ! p ! q ! \zeta !}\left[\omega\left(1-\frac{\mu_{1}}{\mu_{0}}\right) \mathbf{h}_{\alpha p q \zeta}(x, z)\right. \\
& \left.+\omega^{2}\left(\varepsilon_{1}-\varepsilon_{0}\right) \mathbf{e}_{\alpha p q \zeta}(x, z)\right]+O\left(\delta^{n+1}\right),
\end{aligned}
$$

To achieve the proof, we put:

$$
\begin{aligned}
& \mathbf{e}_{1}(\delta ; x, z)=\sum_{|\alpha|=0}^{n+1} \sum_{|p|=0}^{n+1-|\alpha|} \frac{\delta^{|\alpha|+|p|}}{\alpha ! p !} \mathbf{e}_{\alpha p}(x, z), \quad \mathbf{h}_{1}(\delta ; x, z)=\omega \sum_{|\alpha|=0}^{n+1} \sum_{|p|=0}^{n+1-|\alpha|} \frac{\delta^{|\alpha|+|p|}}{\alpha ! p !} \mathbf{h}_{\alpha p}(x, z), \\
& \mathbf{e}_{2}(\delta ; x, z)=\sum_{|\alpha|=0}^{n+1} \sum_{|p|=0}^{n+1-|\alpha|} \sum_{|q|=0}^{n+1-|\alpha|-|p|} \frac{\delta^{|\alpha|+|p|+|q|}}{\alpha ! p ! q !} \mathbf{e}_{\alpha p q}(x, z)
\end{aligned}
$$

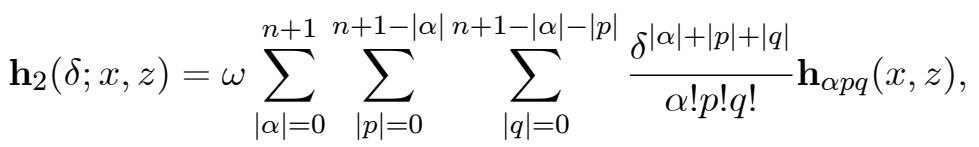

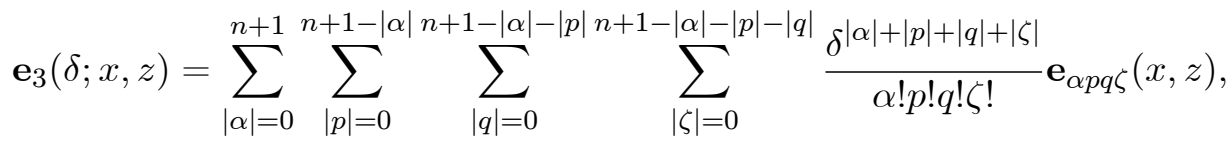

and

$$
\mathbf{h}_{3}(\delta ; x, z)=\omega \sum_{|\alpha|=0}^{n+1} \sum_{|p|=0}^{n+1-|\alpha|} \sum_{|q|=0}^{n+1-|\alpha|-|p|} \sum_{|\zeta|=0}^{n+1-|\alpha|-|p|-|q|} \frac{\delta^{|\alpha|+|p|+|q|+|\zeta|}}{\alpha ! p ! q ! \zeta !} \mathbf{h}_{\alpha p q \zeta}(x, z) .
$$


For $d=2$ and $n=1$, we obtain the following uniform expansion at order 2 .

Corollary 4.7 Let $d=2$ and suppose that we have all hypothesis of Theorem 4.6. Then, the following uniform asymptotic expansion for $x \in \Omega$ holds:

$$
\begin{aligned}
\mathbf{E}_{\delta}(x)= & \mathbf{E}_{0}(x)-\delta^{2}\left\{\left(1-\frac{\mu_{1}}{\mu_{0}}\right) \frac{i}{\mu_{1}} \nabla_{x} \times\left[\nabla_{z} \times \mathbf{G}^{\mathbf{0}}(x, z) \int_{B}\left\{k \Phi_{k}(t, z) \int_{\partial B} \mathbf{j}_{0}(s) d \sigma(s)\right.\right.\right. \\
& \left.\left.+1 / k \nabla \Phi_{k}(t, z) \int_{\partial B} \partial B\left(\mathbf{j}_{0}\right)(s) d \sigma(s)\right\}(t) d t\right] \\
& +\omega^{2}\left({ }_{1}-{ }_{0}\right) \mathbf{G}^{\mathbf{0}}(x, z) \int_{B}\left\{k \Phi_{k}(t, z) \int_{\partial B} \mathbf{j}_{0}(s) d \sigma(s)\right. \\
& \left.\left.+1 / k \nabla \Phi_{k}(t, z) \int_{\partial B} \partial B\left(\mathbf{j}_{0}\right)(s) d \sigma(s)\right\}(t) d t\right\}+O\left(\delta^{2}\right) .
\end{aligned}
$$

where the term $O\left(\delta^{2}\right)$ is dominated by $C \delta^{2}\|\mathbf{g}\|_{T H_{\text {div }}^{-1 / 2}(\partial \Omega)}$ for some positive constant $C$ independent of the geometry of the medium .

\section{References}

[1] H. Ammari and H. Kang, Boundary Layer Techniques for Solving the Helmholtz Equation in the Presence of Small Inhomogeneities, J. Math. Anal. Appl., 296 (2004), no.1, 190-208.

[2] H. Ammari and A. Khelifi, Electromagnetic Scattering by Small Dielectric Inhomogeneities. Journal de Mathématiques Pures et Appliquées 82 (2003), 749-842.

[3] H. Ammari, and D. Volkov, Asymptotic formulas for perturbations in the eigenfrequencies of the full Maxwell equations due to the presence of imperfections of small diameter. Asymptotic Analysis 30 (2002), 331-350.

[4] H. Ammari, M. Vogelius, and D. Volkov, Asymptotic formulas for perturbations in the electromagnetic fields due to the presence of imperfections of small diameter II. The full Maxwell equations, J. Math. Pures Appl. 80 (2001), 769814 .

[5] M. Asch and S. M. Mefire, Numerical localisation of electromagnetic imperfections from a perturbation formula in three dimensions. Journal of Computational Mathematics, Vol.26, No.2, 2008, 149-195.

[6] A. Buffa, P. Ciarlet, Jr. On traces for functional spaces related to Maxwell's equations Part I: An integration by parts formula in Lipschitz polyhedra, Math. Meth. Appl. Sci. 2001; 24:9-30.

[7] A. Buffa, M. Costabel, and C. Schwab, Boundary element methods for Maxwell's equations on non-smooth domains, Numer. Math., 92 (2002), pp. 679-710. 
[8] A. Buffa, M. Costabel, and D. Sheen, On traces for $H(\operatorname{curl}, \Omega)$ in Lipschitz domains, J. Math. Anal. Appl., 276 (2002), pp. 845-867.

[9] D. L. Colton and R. Kress, Integral equation methods in scattering theory, Pure and Applied Mathematics (New York), John Wiley \& Sons Inc., New York, 1983. A Wiley-Interscience Publication.

[10] M. Costabel, Boundary integral operators on Lipschitz domains: Elementary results. SIAM J. Math. Anal. 19(3), 613-626 (1988).

[11] M. Costabel, E. Darrigrand, and H. Kone, Volume and surface integral equations for electromagnetic scattering by a dielectric body, J. Comput. Appl. Math. 234 (2010) 1817-1825.

[12] M. Costabel and F. Le Louër, On the Kleinman-Martin integral equation method for the electromagnetic scattering problem by a dielectric, Proc. 9th International Conference on Mathematical and Numerical Aspects of Wave Propagation, (2009).

[13] Costabel and E. P. Stephan, Strongly elliptic boundary integral equations for electromagnetic transmission problems, Proc. Roy. Soc. Edinburgh Sect. A, 109 (1988), pp. 271-296.

[14] A. Friedman and M. Vogelius, Identification of small inhomogeneities of extreme conductivity by boundary measurements: a theorem on continuous dependence, Arch. Rat. Mech. Anal. 105 (1989), 299-326.

[15] H. Kang and J.K. Seo, Layer potential technique for the inverse conductivity problem, Inverse Problems 12 (1996), 267-278.

[16] D. V. Korikov, Asymptotic of Maxwell system eigenvalues in a domain with small cavities. Algebra i Analiz, 31(1), (2019), pp. 18-71.

[17] D. Mitrea, M. Mitrea and J. Pipher, Vector potential theory on nonsmooth domains in R3 and applications to electromagnetic scattering. J. Fourier Anal. Appl. 3(2), 131-192 (1997).

[18] J. -C. Nédélec, Acoustic and electromagnetic equations, vol. 144 of Applied Mathematical Sciences, Springer-Verlag, New York, 2001. Integral representations for harmonic problems.

[19] G.C. Verchota, Layer potentials and boundary value problems for Laplace's equation in Lipschitz domains, J. of Functional Analysis 59 (1984), 572-611.

[20] M. Vogelius and D. Volkov, Asymptotic formulas for perturbations in the electromagnetic fields due to the presence of inhomogeneities, Math. Model. Numer. Anal. 34 (2000), 723-748. 
[21] I. Terrasse., Résolution mathématique et numérique des équations de Maxwell instationnaires par une méthode de potentiels retardés. Thèse de l'Ecole Polytechnique, CMAP, 91128 Palaiseau Cedex, France, 1993. 